\title{
Homogeneous abundance analysis of FGK dwarf, subgiant, and giant stars with and without giant planets ${ }^{\star}, \star \star$
}

\author{
Ronaldo da Silva ${ }^{1,2}$, André de C. Milone ${ }^{1}$, and Helio J. Rocha-Pinto ${ }^{3}$
}

\author{
1 Divisão de Astrofisíca, Instituto Nacional de Pesquisas Espaciais, São José dos Campos, SP 12201-970, São Paulo, Brazil \\ 2 Dipartimento di Fisica, Università di Roma Tor Vergata, 00133 Rome, Italy \\ e-mail: ronaldo.dasilva@roma2 .infn.it \\ ${ }^{3}$ Observatório do Valongo, Universidade Federal do Rio de Janeiro, 21941-901 Rio de Janeiro, Brazil
}

Received 30 January 2015 / Accepted 7 May 2015

\begin{abstract}
Aims. We have analyzed high-resolution and high signal-to-noise-ratio optical spectra of nearby FGK stars with and without detected giant planets in order to homogeneously measure their photospheric parameters, mass, age, and the abundances of volatile (C, N, and $\mathrm{O}$ ) and refractory ( $\mathrm{Na}, \mathrm{Mg}, \mathrm{Si}, \mathrm{Ca}, \mathrm{Ti}, \mathrm{V}, \mathrm{Mn}, \mathrm{Fe}, \mathrm{Ni}, \mathrm{Cu}$, and $\mathrm{Ba}$ ) elements. Our sample contains 309 stars from the solar neighborhood (up to the distance of $100 \mathrm{pc}$ ), out of which 140 are dwarfs, 29 are subgiants, and 140 are giants.

Methods. The photospheric parameters are derived from the equivalent widths of Fe I and Fe II lines. Masses and ages come from the interpolation in evolutionary tracks and isochrones on the Hertzsprung-Russell diagram. The abundance determination is based on the equivalent widths of selected atomic lines of the refractory elements and on the spectral synthesis of $\mathrm{C}_{2}, \mathrm{CN}, \mathrm{C}$ I, O I, and Na I features. We apply a set of statistical methods to analyze the abundances derived for the three subsamples.

Results. Our results show that: i) giant stars systematically exhibit underabundance in $[\mathrm{C} / \mathrm{Fe}]$ and overabundance in $[\mathrm{N} / \mathrm{Fe}]$ and $[\mathrm{Na} / \mathrm{Fe}]$ in comparison with dwarfs, a result that is normally attributed to evolution-induced mixing processes in the envelope of evolved stars; ii) for solar analogs alone, the abundance trends with the condensation temperature of the elements are correlated with age and anticorrelated with the surface gravity, which agrees with recent studies; iii) as in the case of [Fe/H], dwarf stars with giant planets are systematically enriched in $[\mathrm{X} / \mathrm{H}]$ for all the analyzed elements, except for $\mathrm{O}$ and $\mathrm{Ba}$ (the former due to limitations of statistics), confirming previous findings in the literature that it is not only iron that has an important relation with the planetary formation; and iv) giant planet hosts are also significantly overabundant for the same metallicity when the elements from $\mathrm{Mg}$ to $\mathrm{Cu}$ are combined.
\end{abstract}

Key words. stars: fundamental parameters - stars: abundances - planetary systems - methods: data analysis - methods: statistical techniques: spectroscopic

\section{Introduction}

That stars hosting a giant planet are, on average, more abundant in iron than stars in the solar neighborhood for which no planet has been detected (Santos et al. 2001, 2004; Fischer \& Valenti 2005; Gonzalez 2006) is very accepted. Actually, it has been shown that this behavior is not exclusive to iron but is shared by several other metals (Bond et al. 2006; Gilli et al. 2006; Neves et al. 2009; Adibekyan et al. 2012b). Other studies also suggest that this kind of anomaly may involve not only the metal content of heavy elements but also some light elements such as carbon, nitrogen, and oxygen (Ecuvillon et al. 2004, 2006; Petigura \& Marcy 2011).

Some authors have also been searching for differences in the abundance trends with metallicity for several elements in stars with and without planets. In other words, those works investigated whether the planetary formation affects the observed abundances in some way by comparing stars in the same metallicity range. Robinson et al. (2006) report that the $[\mathrm{Si} / \mathrm{Fe}]$ and $[\mathrm{Ni} / \mathrm{Fe}]$ abundance ratios in planet-host stars are systematically

* Based on public data from the ELODIE archive (Moultaka et al. 2004, http://atlas.obs-hp.fr/elodie/)

$\star \star$ Tables 2-8 are only available at the CDS via anonymous ftp to cdsarc.u-strasbg.fr (130.79.128.5) or via

http://cdsarc.u-strasbg.fr/viz-bin/qcat?]/A+A/580/A24 enhanced over their comparison sample of stars without planets of the same metallicity. Delgado Mena et al. (2010) derived the opposite behavior for $\mathrm{Mg}$, and they find no differences regarding $[\mathrm{C} / \mathrm{Fe}],[\mathrm{O} / \mathrm{Fe}]$, and $[\mathrm{Si} / \mathrm{Fe}]$. In any case, they state that their result for $\mathrm{Mg}$ disappears when only solar analogs are considered. Neves et al. (2009) also report no significant differences in $[\mathrm{X} / \mathrm{Fe}]$ for 12 elements in stars with and without planets. Gonzalez \& Laws (2007) derived lower $[\mathrm{Al} / \mathrm{Fe}]$ and $[\mathrm{Si} / \mathrm{Fe}]$ but higher $[\mathrm{Ti} / \mathrm{Fe}]$ in stars with planets in the range of metal-rich stars. Brugamyer et al. (2011), on the other hand, confirm the $[\mathrm{Si} / \mathrm{Fe}]$ enhancements in planet hosts, in spite of no $[\mathrm{O} / \mathrm{Fe}]$ enhancements. Overabundances of several elements were also reported by Kang et al. (2011) and Adibekyan et al. (2012a), but only in the range of metal-poor stars, except for Mn. For this element, Kang et al. (2011) find an enhancement in the whole range of metallicity, a result that is not supported by Adibekyan et al. (2012a). The situation is, therefore, vast and varied, and also controversial for some elements.

In a previous paper (da Silva et al. 2011, from now on referred to as Paper I), we determined photospheric parameters and carbon abundances for a sample of 172 dwarf, subgiant, and giant stars (out of which 18 have planets) using spectra available in the ELODIE database (Moultaka et al. 2004). Our previous results did not point out, for instance, any significant difference between the carbon abundances of stars with and without planets, 
for the three substellar groups. However, the analyzed sample was too small to provide conclusive results, and it stimulated the inclusion of additional stars and other chemical elements.

In the current work we have 309 FGK stars (out of which 47 have detected planets) homogeneously analyzed to measure their photospheric parameters, masses, ages, and the abundances of volatile $(\mathrm{C}, \mathrm{N}$, and $\mathrm{O})$ and refractory $(\mathrm{Na}, \mathrm{Mg}, \mathrm{Si}, \mathrm{Ca}, \mathrm{Ti}, \mathrm{V}, \mathrm{Mn}$, $\mathrm{Fe}, \mathrm{Ni}, \mathrm{Cu}$, and $\mathrm{Ba}$ ) elements. The sample includes dwarf, subgiant, and giant stars, mostly belonging to the Galactic thin disk. We applied a set of statistical methods to the three subsamples, exploring their multivariate properties and, in particular, uncovering significant differences between dwarf stars with and without a known giant planet. In addition, we searched for relations involving the stellar parameters, the abundances, and the condensation temperature of the elements. This approach has very often been used to check whether any difference in the amount of refractory elements with respect to volatile ones has an influence on the formation of planetary systems (see, e.g., Smith et al. 2001; Meléndez et al. 2009; Ramirez et al. 2009; González Hernández et al. 2010, 2013a,b).

In Sect. 2 we describe the observations and the procedure of data reduction. In Sect. 3 we outline the methods used to derive the photospheric parameters and the chemical abundances. The determination of the evolutionary and the kinematic parameters is presented in Sect. 4. The results are presented and discussed in Sects. 5 and 6, in which we describe the statistical analysis. Finally, we present our final remarks and conclusions in Sect. 7.

\section{Observation data and reduction}

In this work, as a complement to our previous analysis of 172 stars published in Paper I, we have enlarged our stellar sample. Now we have 309 FGK stars from the solar neighborhood that include 140 dwarfs (31 of which have detected planets), 29 subgiants (4 with planets), and 140 giants (12 with planets).

The stars were observed with the ELODIE high-resolution spectrograph (Baranne et al. 1996) of the Haute Provence Observatory (France), and the data are publicly available in the online ELODIE database. The ELODIE spectrograph provides spectra with resolution $R \sim 42000$ in the wavelength range 3895-6815 A. The selection criteria are similar, but not equal, to those applied before since we have now considered a smaller inferior limit for the signal-to-noise ratio $(\mathrm{S} / \mathrm{N})$. Following this new criteria we selected:

i) stars for which the averaged spectra have $S / N \geq 150$ : only individual spectra with a $S / N \geq 20$ and with an image type classified as object fiber only (OBJO) were used;

ii) stars within a distance $\leq 100 \mathrm{pc}$ (parallax $\pi \geq 10$ mas) and with spectral type between F8 and M1;

iii) stars having no close-in binary companion that could contaminate the observed spectra: we used the information of the angular separation between components (rho) available in the HIPPARCos catalog (ESA 1997), and we chose only those systems with $r h o>10$ arcsec; additionally, we searched in literature for any further information that could unveil a contaminant star in the observed field;

iv) stars with $(B-V)$ measured by HIPPARCos and with crosscorrelation parameters available in the ELODIE database: both $(B-V)$ and the width of the spectral cross-correlation function are required to carry out a first estimate of the projected rotation velocity vsin $i$ of each star; and v) stars for which the determination of the photospheric parameters is reliable (see Sect. 3.1) and that passed the quality control of the spectral synthesis (see Sect. 3.2).

A total of 1485 spectra of 309 stars were analyzed. The subsamples of dwarfs, subgiants, and giants are plotted in the Hertzsprung-Russell (HR) and color-distance diagrams of Fig. 1. As in Paper I, we chose to classify as subgiants those stars situated 1.5 mag above the lower limit of the main sequence and with $M_{\mathrm{v}}>2.0 \mathrm{mag}$. The distance of dwarfs and subgiants is not limited to $100 \mathrm{pc}$, but to about $80 \mathrm{pc}$, which represents a selection effect of the ELODIE observation surveys. For comparison, in the righthand panel of this figure we plot the $\log g$ vs. $T_{\text {eff }}$ diagram with the stellar parameters derived in the current work.

The spectra were reduced using IRAF $^{1}$ routines for order identification and extraction, background subtraction, flat-field correction, wavelength calibration, radial-velocity shift correction, and flux normalization (a global normalization first, and then a more careful one performed around regions containing molecular bands and atomic lines used in the spectral synthesis).

\section{Spectroscopic analysis}

\subsection{Photospheric parameters and equivalent widths}

In this work, as in Paper I, we performed a differential analysis relative to the Sun. The method requires a model atmosphere for each star, which in turn is computed using the so-called photospheric parameters: effective temperature $T_{\text {eff }}$, metallicity $[\mathrm{Fe} / \mathrm{H}]$, surface gravity $\log g$, and microturbulent velocity $\xi$. We computed these parameters through the excitation equilibrium of neutral iron and the ionization equilibrium between Fe I and Fe II lines, using model atmospheres derived by interpolation in the grid of Kurucz (1993). As before, we used the MOOG LTE radiative code (Sneden 2002) and the same routine as developed by us to perform an automated calculation of the parameters and their uncertainties, allowing a fast and homogeneous approach.

To compute the solar model atmosphere, we adopted $T_{\text {eff }}=$ $5777 \mathrm{~K}, \log g=4.44, \xi=1.0 \mathrm{~km} \mathrm{~s}^{-1}$, and the standard solar abundances of Anders \& Grevesse (1989), but with $\log \epsilon_{\odot}(\mathrm{Fe})=$ 7.47 , to be consistent with the value adopted in Paper I. We also assumed the Unsöld approximation multiplied by 6.3 to account for the van der Waals line damping. This is useful for a better treatment of strong lines close to the spectral windows used in our spectral synthesis analysis.

Table 2 shows an excerpt of the atomic line parameters (wavelength, statistical weight of the lower level multiplied by the oscillator strength $g f$, and lower-level excitation potential $\chi$ ) and equivalent width (EW) for our list of atomic lines. Table 3 contains an excerpt of the $g f$ values for elements with important hyperfine structure (HFS). The complete tables for all the elements analyzed in the present work are available in electronic form at the CDS. These parameters were initially taken from the Vienna Atomic Line Database - VALD (Kupka et al. 2000, 1999; Piskunov et al. 1995; Ryabchikova et al. 1997) in the case of the elements without HFS, and from Steffen (1985) for those with significant HFS. The $g f$ values were then revised in order to fit the equivalent widths measured in the spectrum of the Solar Flux Atlas (Kurucz et al. 1984) degraded to the ELODIE resolution. For the Sun and for all the other stars in our sample, the $E W \mathrm{~s}$ were measured using the Automatic Routine for line Equivalent widths in stellar Spectra (ARES, Sousa et al. 2007).

\footnotetext{
1 Image Reduction and Analysis Facility, distributed by the National Optical Astronomy Observatories (NOAO), USA.
} 
R. da Silva et al.: Abundances in dwarfs, subgiants, and giants with and without planets
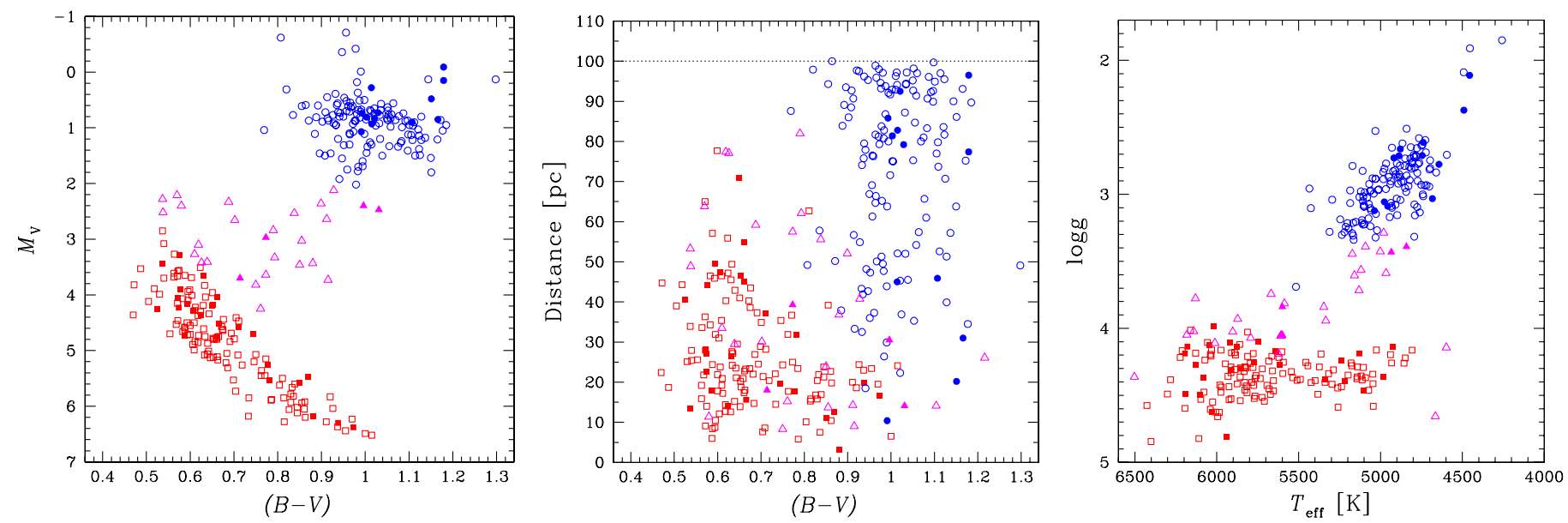

Fig. 1. Hertzsprung-Russell (left panel) and color-distance (middle panel) diagrams for our sample of 140 dwarfs ( $\square$ ), 29 subgiants $(\triangle)$, and 140 giants (O). The derived stellar parameters are also shown in a $\log g$ vs. $T_{\text {eff }}$ diagram (right panel) for comparison. Filled symbols represent stars with detected planets. The distance limit of $100 \mathrm{pc}$ is represented by the dotted line.

The atomic lines of the elements $\mathrm{Mg}, \mathrm{V}, \mathrm{Mn}$, and $\mathrm{Cu}$ have significant HFS that has to be taken into account. In the case of those elements with lines not listed in Steffen (1985), we adopted the values of neighboring multiplets. An exception is the $\mathrm{Mg}$ line at $\lambda 5785.29$, for which no HSF information was available. Its $g f$ value was obtained in the same way as those lines without HFS listed in Table 2. This line is not strong $(E W<60 \mathrm{~m} \AA)$, and the error induced in the $\mathrm{Mg}$ abundance determination is of second order. The HFS of $\mathrm{Ba}$ and also its isotopic splitting are of some significance but only for the line at $\lambda 6496.91$, because they are negligible for the lines at $\lambda 5853.69$ and $\lambda 6141.73$ (see Korotin et al. 2011).

Table 4 shows an excerpt of the derive photospheric parameters for the 140 dwarf stars in our sample, along with their uncertainties. The complete tables, also for subdwarfs and giants, are available in electronic form at the CDS.

\subsection{Abundance determination}

The abundances of the refractory elements ( $\mathrm{Na}, \mathrm{Mg}, \mathrm{Si}, \mathrm{Ca}, \mathrm{Ti}$, $\mathrm{V}, \mathrm{Mn}, \mathrm{Ni}, \mathrm{Cu}$, and $\mathrm{Ba}$ ), with respect to $\mathrm{Fe}$, were determined based on the equivalent widths of a selection of spectral lines chosen as free from blends by inspection of the Solar Flux Atlas and the solar line identification catalog of Moore et al. (1966).

We used the abfind (in the case of elements without important HFS) and the blends (in the case of elements with HFS) drivers of the MOOG code to derive the abundances. These drivers require (i) the model atmospheres, which were previously computed using the photospheric parameters derived for each star; and (ii) a list of the selected lines, containing the respective wavelength (or the hyperfine structure), the atomic number, the lower-level excitation potential, the revised values of $g f$, and the measured equivalent widths. Again, using another routine for an automated calculation developed by us, we derived the abundances for each element in turn, excluding (by means of a sigma-clipping procedure) spectral lines that provided an incorrect abundance determination.

For the volatile elements (C, N, and $\mathrm{O})$ and for $\mathrm{Na}$ as well, we used the spectral synthesis technique, also performed with the MOOG code, to reproduce the observed spectra in regions containing molecular lines of electronic-vibrational band heads of the $C_{2}$ Swan system (centered at $\lambda 5128$ and 25165 ), of the $\mathrm{CN}$ blue system (centered at 24215 ), and to atomic lines of
$\mathrm{C}(\lambda 5380.34), \mathrm{O}(\lambda 6300.30)$, and $\mathrm{Na}(\lambda 6154.23$ and $\lambda 6160.75)$. Again, as in Paper I, we preferred not to use the $\mathrm{C}$ atomic line at $\lambda 5052.17$ because it is blended with a strong Fe line, which may affect the abundance determination.

The synthetic spectra were computed in wavelength steps of $0.01 \AA$, also considering the continuum opacity contribution in ranges of $0.5 \AA$, line-broadening corrections (from velocity fields and instrumental broadening), and the limb darkening effect. Some parameters of atomic and molecular lines were taken from the VALD database and from Kurucz (1995). Additional molecules that contribute to the spectral line formation in the studied wavelength regions are $\mathrm{MgH}$ (at $\lambda 5128$, $\lambda 5165$, and for the $\mathrm{C}$ atomic line), $\mathrm{CH}$ (at $\lambda 4215$ ), and $\mathrm{CN}$ (for the $\mathrm{Na}$ and $\mathrm{O}$ atomic lines), though their contributions are relatively small. The $g f$ values of some atomic and molecular lines were revised to fit the solar spectrum, which was taken as a reference in our differential chemical analysis. The oxygen line at $\lambda 6300.30$ is blended with a Ni line at $\lambda 6300.34(\log g f=$ $-2.3098)$. Therefore, we derived the $\mathrm{O}$ abundances by taking the $\mathrm{Ni}$ abundance of each star into account when fitting the observed profile. Moreover, to account for the presence of telluric lines in this region, we adopted the telluric spectrum by Wallace et al. (2011) with the resolution degraded to match our spectra.

To account for spectral line broadening from velocity fields, as in Paper I, we adopted the same definition of $V_{\text {broad }}$ (also listed in Table 4), which is a composite of velocity fields, such as rotation and macro-turbulent velocities. The instrumental broadening was estimated from the width of thorium lines, and the linear coefficient $u$ of the stellar limb darkening was taken from Díaz-Cordovés (1995).

Figure 2 shows the spectral synthesis applied to three of the regions used for abundance determination, the $C_{2}(\lambda 5165)$ and $\mathrm{CN}$ ( 24215$)$ molecular band heads and the $\mathrm{O}$ atomic line at $\lambda 6300.30$, for two dwarf stars with different spectral $\mathrm{S} / \mathrm{N}$. The synthetic spectra were resampled in steps of $0.05 \AA$ to consistently match the wavelength scale of the observed spectra. For examples of spectral synthesis applied to other regions and to other stars, we refer the reader to Figs. 3 and 5 of Paper I.

For a comparison between the results provided by molecularand atomic-line $\mathrm{C}$ abundances, Fig. 3 shows the differences plotted as a function of $T_{\text {eff }}$ and of $\mathrm{S} / \mathrm{N}$ for our subsamples of dwarfs, subgiants, and giants. Besides a slight underabundance (within errors, however) of molecular with respect to atomic 

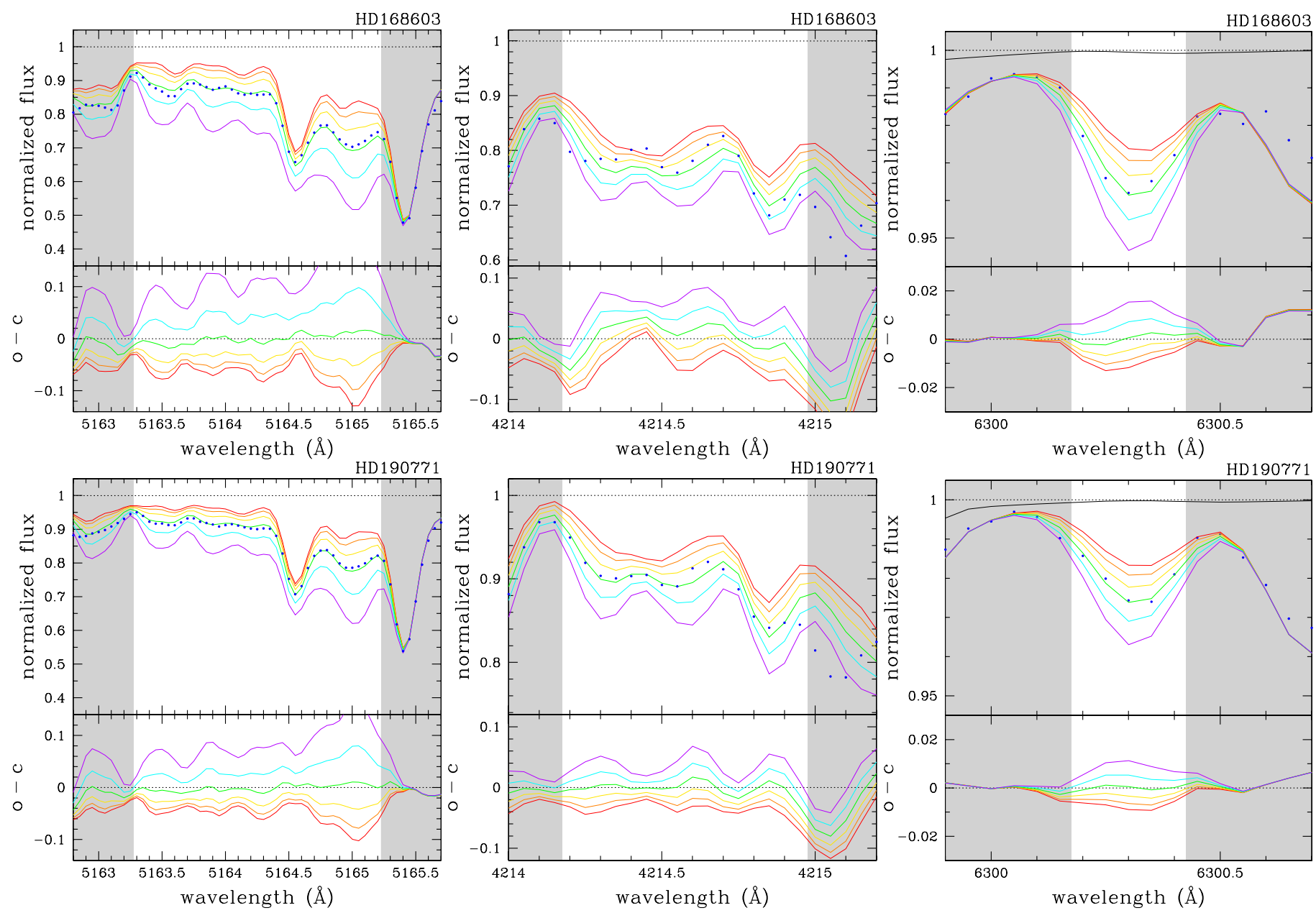

Fig. 2. Synthesis of three spectral regions: $\mathrm{C}_{2}(\lambda 5165$, left panels $)$ and $\mathrm{CN}(\lambda 4215$, middle panels $)$ molecular band heads, and the $\mathrm{O}$ atomic line at $\lambda 6300.30$ (right panels). Two dwarf stars with different values of spectral $\mathrm{S} / \mathrm{N}$ are shown: HD $168603\left(S / N=156, T_{\mathrm{eff}}=5399 \mathrm{~K},[\mathrm{Fe} / \mathrm{H}]=\right.$ 0.11 dex, top panels $)$ and $\mathrm{HD} 190771\left(S / N=299, T_{\text {eff }}=5817 \mathrm{~K},[\mathrm{Fe} / \mathrm{H}]=0.14 \mathrm{dex}\right.$, bottom panels $)$. These are the $\mathrm{S} / \mathrm{N}$ per pixel computed at 15550. Six spectra computed for different abundances (the $\mathrm{N}$ abundance is changed in the case of the $C N$ band) and separated by 0.1 dex are plotted. The differences between observed and computed spectra $(\mathrm{O}-\mathrm{C})$ are also plotted in the bottom of each panel.
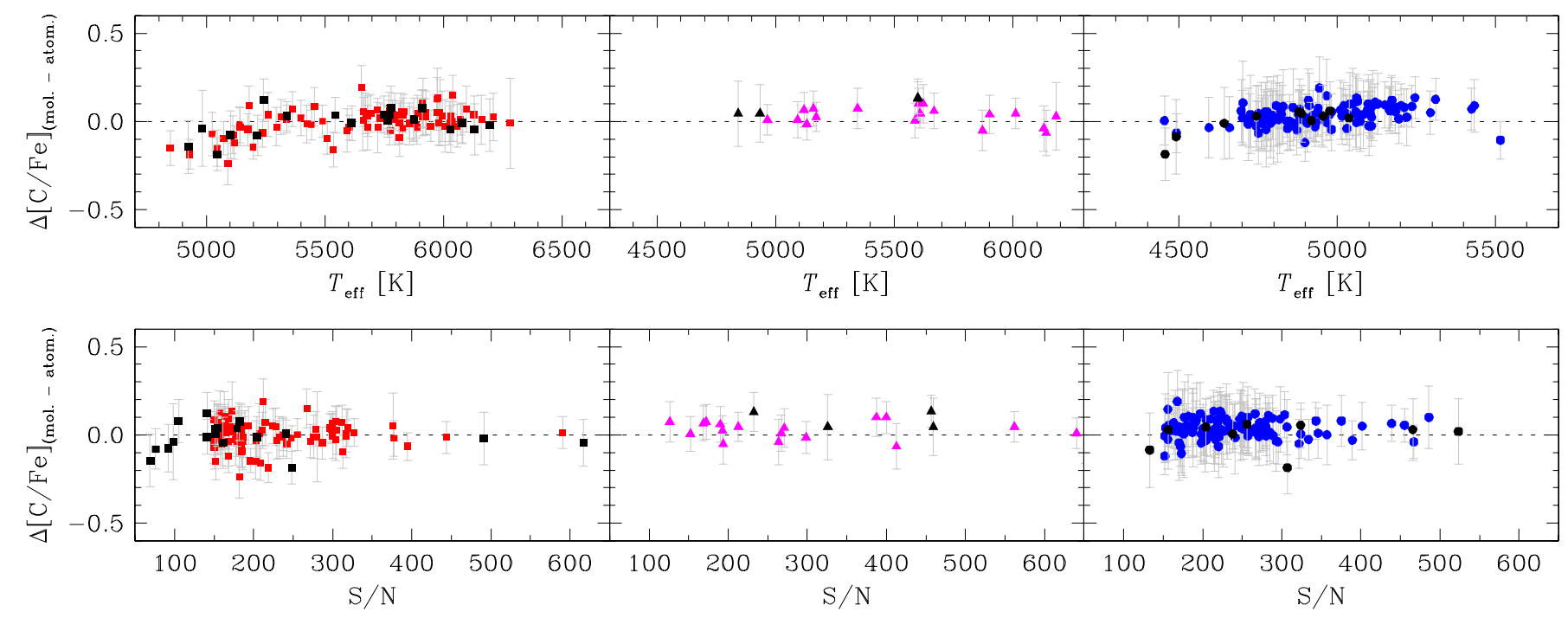

Fig. 3. Difference between the $C$ abundances derived from the $C_{2}$ molecular bands $(\lambda 5128$ and $\lambda 5165)$ and the $C$ atomic line $(\lambda 5380.34)$ as a function of the effective temperature (top panels) and of the $\mathrm{S} / \mathrm{N}$ (bottom panels) for our sample of dwarfs (left panels), subgiants (middle panels), and giants (right panels). Stars with planets are represented by black symbols. 

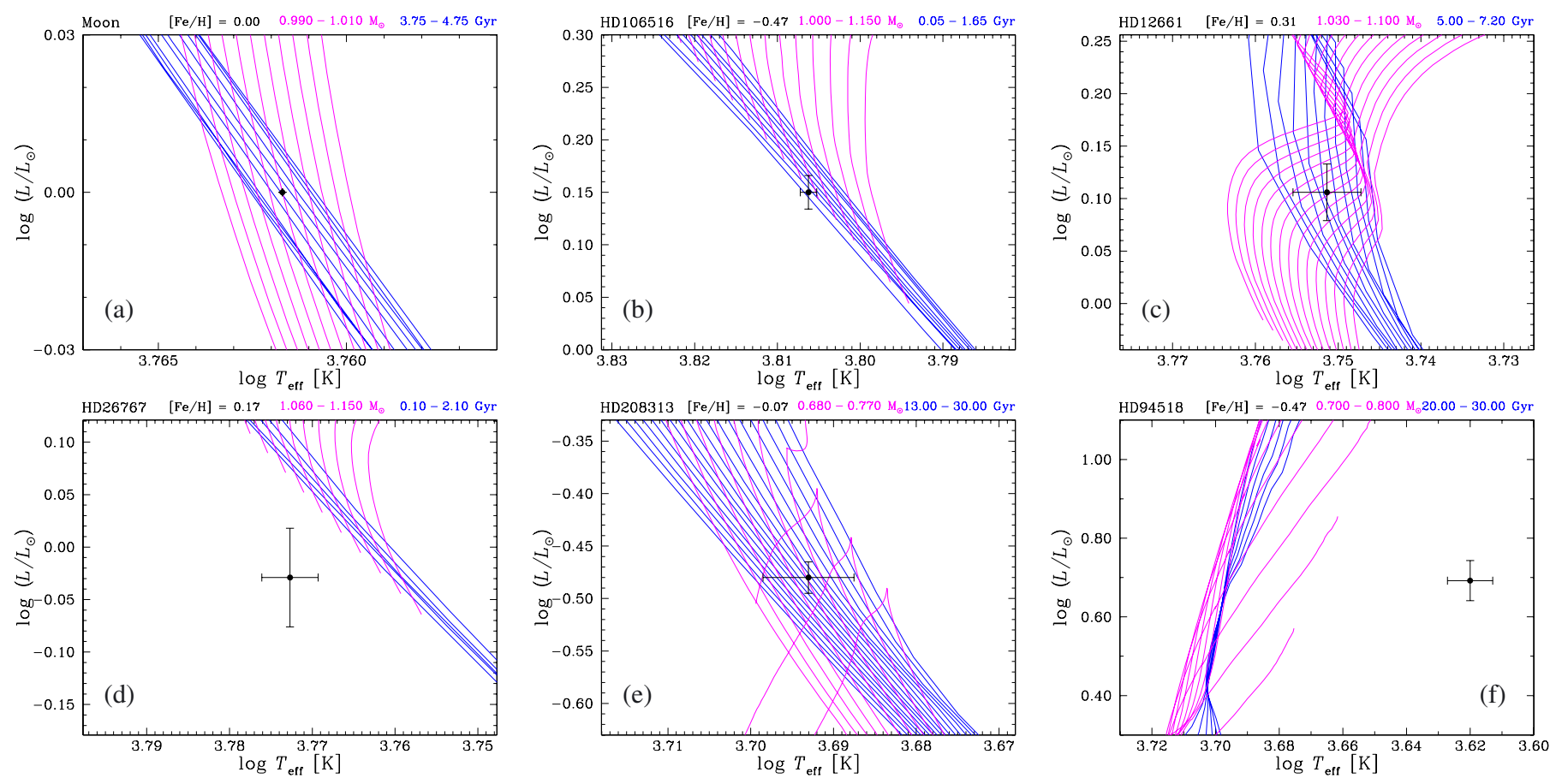

Fig. 4. Evolutionary tracks (magenta lines) and isochrones (blue lines) from Yonsei-Yale, calculated for different values of metallicity, showing how we derived the stellar masses and ages. The ranges of age (increasing from left to right, HD 94518 excepted) and mass (increasing from right to left, HD 94518 excepted) of the models plotted are indicated on the top of each panel.

C abundances for cool dwarfs, no systematic difference is observed in the whole range of $T_{\text {eff }}$ or $\mathrm{S} / \mathrm{N}$.

We estimated the uncertainties in the same way as in Paper I, i.e., using our routine that includes the error propagation of the input parameters used by MOOG: the photospheric parameters $T_{\text {eff }}, \log g,[\mathrm{Fe} / \mathrm{H}]$, and $\xi$, and the broadening velocity $V_{\text {broad }}$. For each one in turn, the input parameters are iteratively changed by their errors, and new values of abundance are computed. The difference between new and best determination gives the error due to each parameter uncertainty, and $\sigma([\mathrm{X} / \mathrm{Fe}])$ is a quadratic sum of individual contributions. The error in $V_{\text {broad }}$ was estimated to be of the order of $1 \mathrm{~km} \mathrm{~s}^{-1}$ or smaller.

Table 4 shows an excerpt with the derived [Ti/Fe] abundance ratios for the 140 dwarf stars. The complete tables with abundances of all the other elements, also for subdwarfs and giants, are available in electronic form at the CDS. The parameters of atomic and molecular lines are listed in Tables 5 and 6, which are only available in electronic form at the CDS as well. Table 5 contains the wavelength of the spectral feature, the atomic and molecular line identification, the lower-level excitation potential, the $g f$ values, and the dissociation energy $D_{0}$ (for molecular features alone). Table 6 (strong atomic lines) contains the same information as in Table 5, except the dissociation energy parameter.

\section{Evolutionary and kinematic parameters}

\subsection{Stellar masses and ages}

We derived masses and ages through interpolations in the grids of Yonsei-Yale $\left(\mathrm{Y}^{2}\right)$ evolutionary tracks and isochrones (Yi et al. 2001; Kim et al. 2002) on the HR diagram. The determination of these parameters takes the metallicity of each star into account. The stars' luminosities used in the diagrams were calculated with parallaxes taken from the new reduction of the HIPPARCos data (van Leeuwen 2007), and bolometric corrections (BC) from Flower (1996), which were revised by Torres (2010). For the Sun, we adopt $B C_{\odot}=-0.07$ and $M_{\text {bol }}^{\odot}=4.75$.

A few examples for different metallicities are illustrated in Fig. 4. To reproduce the Sun's position in the HR diagrams, adopting $T_{\text {eff }}=5777 \mathrm{~K}$ and age $=4.53$ Gy (Guenther \& Demarque 1997), the evolutionary tracks and isochrones were displaced in $\log \left(T_{\text {eff }}\right)$ by $0.001628\left(\sim 22 \mathrm{~K}\right.$ in $\left.T_{\text {eff }}\right)$ and in $\log \left(L / L_{\odot}\right)$ by 0.011 . By doing so, we derived a mass $M=$ $1.003 \pm 0.004 M_{\odot}$ and an age $=4.37 \pm 0.17$ Gyr (see Fig. 4a) for the Sun. The figure also shows a metal-poor and young star (HD 106516, Fig. 4b); a metal-rich one (HD 12661, Fig. 4c); a young star that is outside the lower border of the models (HD 26767, Fig. 4d); a low-mass and old star (HD 208313, Fig. 4e); and another star outside the limits of the models, this time in the region of low-mass and old stars (HD 94518, Fig. 4f).

The star HD 26767 is known to be a member of the Hyades cluster with $625 \mathrm{Myr}$, and it has been studied in several works in recent decades. Other stars that are either very young or outside the lower border of the models have been identified as members of the Hyades, the Ursa Major ( 500 Myr), or the Pleiades (20-150 Myr) moving groups, or even classified as possible subdwarfs. In the case of old stars outside the model limits, they are likely to be low-mass stars no older than the age of the Universe (13.8 Gyr, Komatsu et al. 2011).

Table 7 lists the derived masses and ages, along with the parameters used to compute the luminosities. The uncertainties were estimated taking the error propagation of the photospheric parameters and of the luminosity into account, using $\sigma(\pi)$ from HIPPARCOS and adopting $\sigma(V)=0.01$ and $\sigma(B C)=0.005$. In view of the asymmetric distribution of the isochrones on the HR diagram, the table shows the lower and the upper uncertainties on age. 


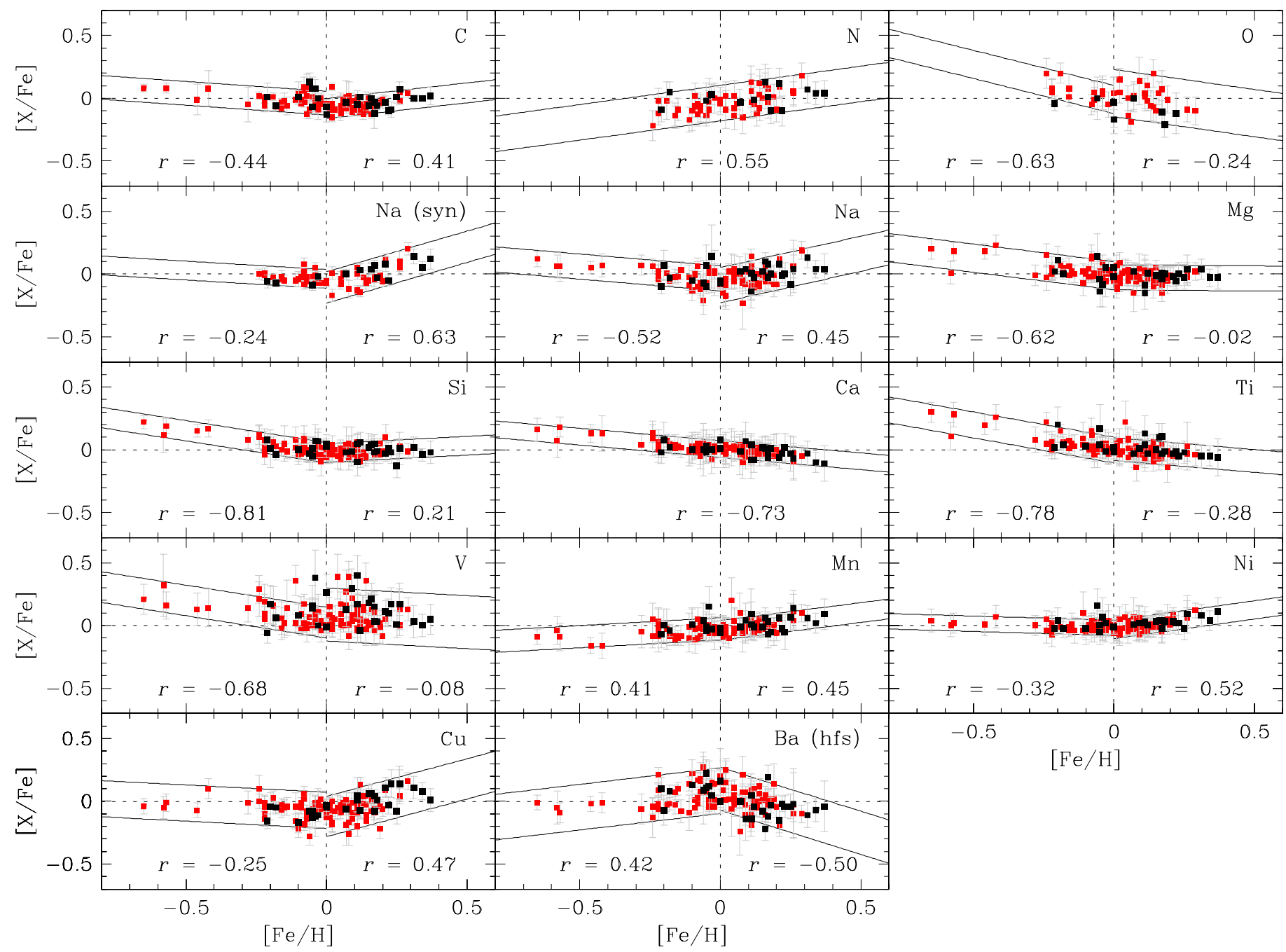

Fig. 5. Abundance ratios as a function of metallicity for our sample of $120 \mathrm{dwarf}$ stars of the thin-disk population. Stars with planets are represented by black symbols. For sodium, the abundance ratios from both spectral synthesis and equivalent widths determination are plotted. The 95\% confidence intervals (solid lines) around linear regressions, and their respective correlation coefficients ( $r$ ) are also shown. The linear regressions were derived either for $[\mathrm{Fe} / \mathrm{H}] \leq 0,[\mathrm{Fe} / \mathrm{H}] \geq 0$, or for the whole metallicity range, depending on which one has the more significant slope.

\subsection{Kinematic properties}

In the same way as in Paper I, we grouped our stars according to their main population membership by computing the probability that a given star belongs to the Galactic thin disk, thick disk, halo, or a population of transition. First, using equations of Johnson \& Soderblom (1987), we computed the space-velocity components $U_{\mathrm{LSR}}, V_{\mathrm{LSR}}$, and $W_{\mathrm{LSR}}$ with respect to the local standard of rest (LSR) using parallaxes and proper motions from the new reduction of HIPPARCOS and radial velocities from the ELODIE archive from the Simbad database and from Holmberg et al. (2007). For the Sun, we adopted $U_{\mathrm{LSR}}=10.0, V_{\mathrm{LSR}}=5.3$, and $W_{\mathrm{LSR}}=7.2 \mathrm{~km} \mathrm{~s}^{-1}$ (Dehnen \& Binney 1998). Then, employing the equations of Reddy et al. (2006), we computed the probability that a star belongs to one of the three populations. A probability $P_{\text {thin }}, P_{\text {thick }}$, or $P_{\text {halo }}$ greater than or equal to $70 \%$ classifies the star as a thin, a thick, or a halo member, respectively. If the probability is in between these, then the star is classified as belonging either to the thin/thick or to the thick/halo transition group.

Our sample of 309 stars contains 281 thin-disk (out of which 120 dwarfs, 25 subgiants, and 136 giants), and 16 thick-disk (10 dwarfs, 2 subgiants, and 4 giants) population members. None of them belongs to the halo, and 13 ( 10 dwarfs, 2 subgiants, and 1 giant) belong to a thin/thick transition population. The membership of each star is indicated in Table 7 .

\section{Results and discussion}

\subsection{Abundance trends with metallicity}

The $[\mathrm{X} / \mathrm{Fe}]$ abundance ratios as a function of metallicity are plotted in Figs. 5-7 for dwarfs, subgiants, and giants, respectively. Since most of our sample stars belong to the thin disk, for homogeneity, only these stars were used in the abundance analysis. They are all listed in the tables, but only thin-disk members are displayed in the figures. Besides iron, titanium is the only element in our study having spectral lines of both neutral and singly ionized atoms. We have found no significant difference between Ti I and Ti II, so we adopted an average value of all lines together.

Despite the small number of metal-poor $([\mathrm{Fe} / \mathrm{H}]<-0.3 \mathrm{dex})$ or metal-rich $([\mathrm{Fe} / \mathrm{H}]>0.3 \mathrm{dex})$ stars in this sample, the trends in the abundance ratios with metallicity are in good agreement with those published in the literature about the nucleosynthetic origin of the elements and the evolution of their abundances with time. 
R. da Silva et al.: Abundances in dwarfs, subgiants, and giants with and without planets

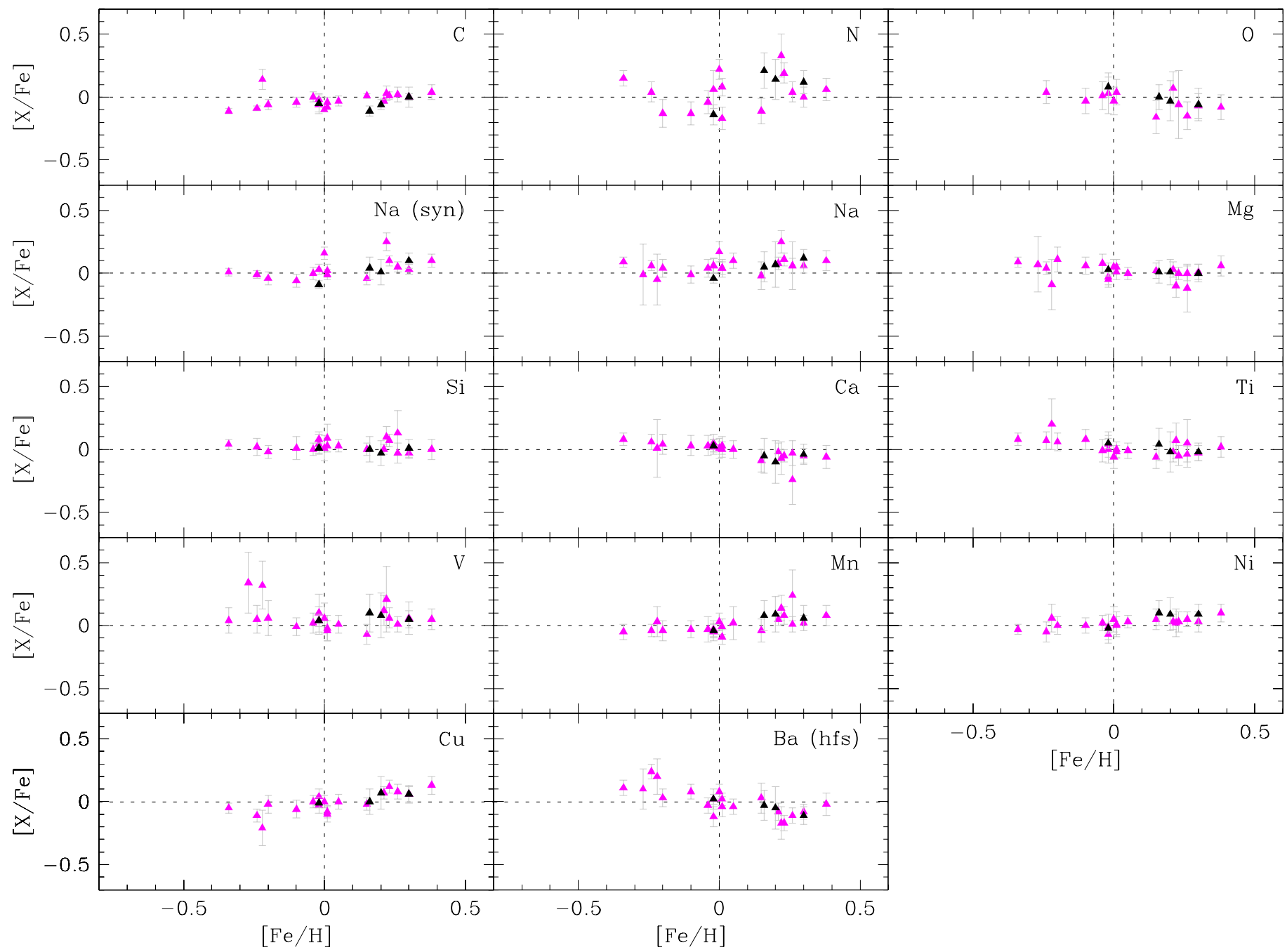

Fig. 6. Same as Fig. 5 but showing the abundance ratios of 25 subgiant stars of the thin disc population.

Regarding the abundance trends for dwarfs, we recommend, for example, Adibekyan et al. (2012b) and Kang et al. (2011). For giant stars see, for example, Takeda et al. (2008). The abundance trends for subgiants are very similar to those for dwarfs for all the investigated elements. A few cases concerning the abundances of the three subsamples deserve particular attention, and we discuss them in the following paragraphs.

There are systematic differences in the abundance ratios of carbon, nitrogen, and sodium when the subsamples of dwarfs, subgiants, and giants are compared with each other: in the range of solar metallicity (from -0.1 to $0.1 \mathrm{dex}$ ) giant stars are underabundant in $[\mathrm{C} / \mathrm{Fe}]$ by $-0.17 \pm 0.07 \mathrm{dex}$ and overabundant in $[\mathrm{N} / \mathrm{Fe}]$ by $0.31 \pm 0.10 \mathrm{dex}$ and in $[\mathrm{Na} / \mathrm{Fe}]$ by $0.16 \pm 0.08 \mathrm{dex}$. The abundance trends for these elements confirm our previous result for carbon discussed in Paper I and agree with the results of Takeda et al. (2008) for sodium and carbon. For oxygen, we found $[\mathrm{O} / \mathrm{Fe}]=0.07 \pm 0.07 \mathrm{dex}$ in the solar metallicity range. Therefore, we do not confirm the oxygen deficiency found by Takeda et al. (2008), who used the forbidden [O I] line at $5577 \AA$. Their oxygen abundances were in either case probably underestimated as affirmed by the authors. Our results also agree with those of Liu et al. (2010), who find that $[\mathrm{C} / \mathrm{Fe}]$ is depleted by $0.13 \mathrm{dex},[\mathrm{Na} / \mathrm{Fe}]$ is overabundant by $0.1 \mathrm{dex}$, and $[\mathrm{O} / \mathrm{Fe}]$ was not altered after the first dredge-up. From a theoretical point of view, changes in the surface abundances of evolved stars is expected for $\mathrm{C}$ and $\mathrm{N}$, and unexpected for $\mathrm{O}$ according to quantitative predictions made by Iben (1991).

A commonly used explanation for such systematic differences is based on the effect of mixing processes that dredge up $\mathrm{C}$-poor and $\mathrm{N}$ - and Na-rich material (produced by $\mathrm{CN}$ and $\mathrm{NeNa}$ cycles) to the surface of evolved stars. On the other hand, the use of spectral lines contaminated by blends is also a possibility. To test this, we derived the sodium abundances using the two techniques described in Sect. 3.2 from the equivalent widths and from spectral synthesis. The available $\mathrm{Na}$ lines in our spectral coverage are affected by $\mathrm{C}_{2}$ and $\mathrm{CN}$ molecular bands. Performing spectral synthesis allows including the already derived $\mathrm{C}$ and $\mathrm{N}$ abundances and, therefore, accounting for the presence of such bands. However, in doing so, the $[\mathrm{Na} / \mathrm{Fe}]$ overabundance in giants is only slightly reduced, so not enough to cancel out the larger systematic difference with respect to dwarfs, thereby favoring the mixing-process hypothesis. Another result that favors this hypothesis is that $[\mathrm{C} / \mathrm{Fe}]$, $[\mathrm{N} / \mathrm{Fe}]$, and $[\mathrm{Na} / \mathrm{Fe}]$ have a trend with mass: a negative trend with increasing mass in the case of carbon and positive ones for nitrogen and sodium (see Fig. 8). More massive stars have evolved faster and, therefore, have become $\mathrm{C}$-poorer and $\mathrm{N}$ - and Na-richer than stars with masses close to the solar value. The oxygen abundances remain constant and independent of mass, supporting the above discussion. 


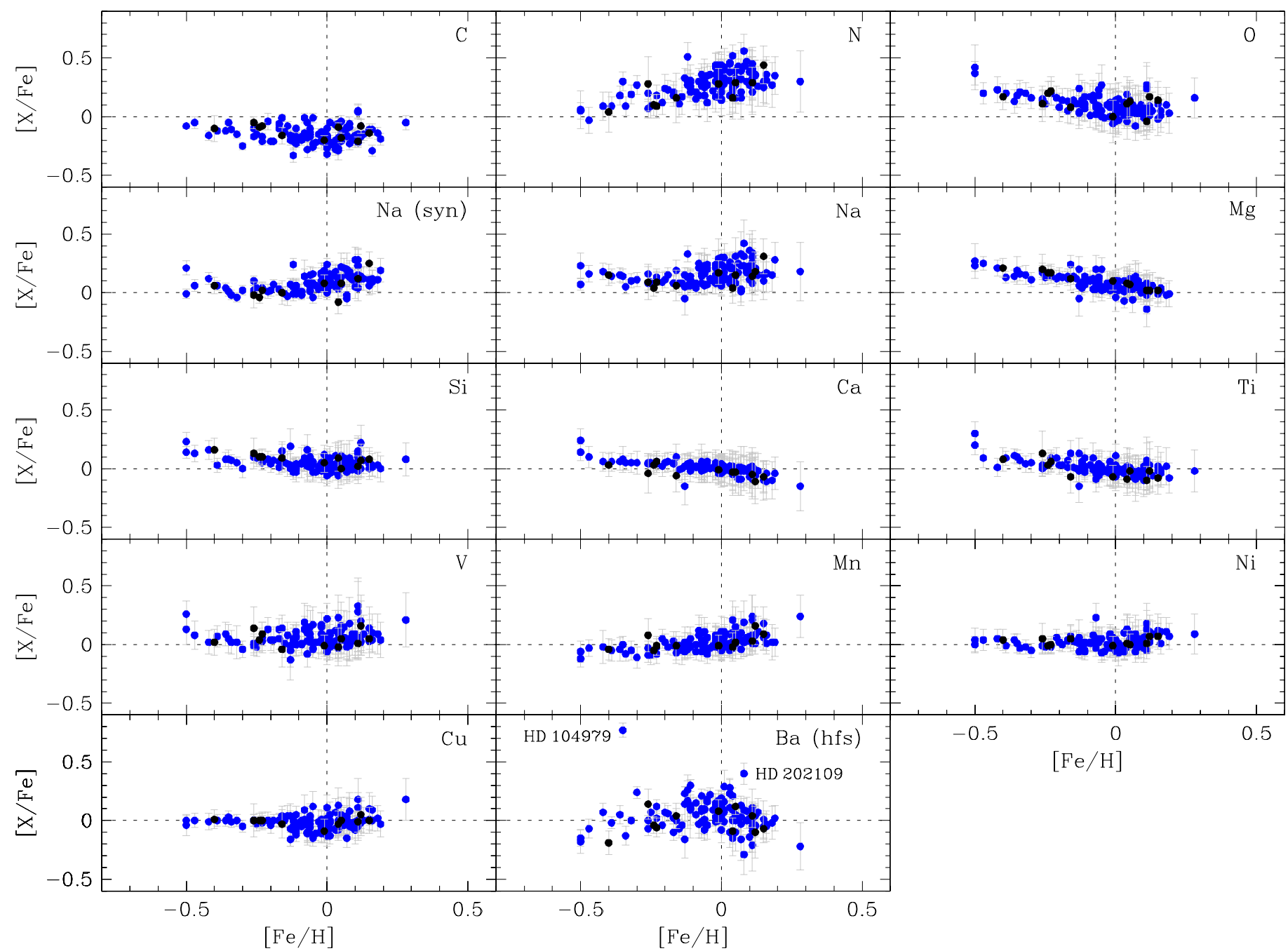

Fig. 7. Same as Fig. 5 but showing the abundance ratios of 136 giant stars of the thin-disk population. The stars indicated are discussed in Sect. 5.1.1.

The nitrogen abundances of our giant stars show a clear dependence on the effective temperature (see Fig. 9, top right panel). One possibility is that this dependence is related to the continuum definition when using the $\mathrm{CN}$ molecular band. In this case, the reader would ask whether the trend in $[\mathrm{N} / \mathrm{Fe}]$ vs. $[\mathrm{Fe} / \mathrm{H}]$ is also related to this dependence on $T_{\text {eff }}$, owing to a bad continuum definition. However, if we limit the effective temperature within the almost flat region seen in the $[\mathrm{N} / \mathrm{Fe}]$ vs. $T_{\text {eff }}$ plane, from about 4600 to $5000 \mathrm{~K}$, the trend in [N/Fe] with metallicity still remains. Moreover, the effective temperature of our sample of giants increases with increasing mass; that is, it seems that more evolved stars have higher temperatures. Therefore, we believe that the $[\mathrm{N} / \mathrm{Fe}]$ dependence on $T_{\text {eff }}$ is probably related to evolution effects instead of to a false continuum definition.

For the carbon abundances in dwarf stars, our result is in line with those of Delgado Mena et al. (2010) in the range of solar metallicities. They found, however, a steeper trend in $[\mathrm{C} / \mathrm{Fe}]$ vs. $[\mathrm{Fe} / \mathrm{H}]$ in the range of metal-poor stars, and practically a flat distribution of metal-rich dwarfs. On the other hand, González Hernández et al. $(2010,2013 b)$ derived $[\mathrm{C} / \mathrm{Fe}]$ ratios that continuously decrease with increasing $[\mathrm{Fe} / \mathrm{H}]$ in the whole range of metallicities for their sample of dwarfs and solar analogs.

For the nitrogen abundances of dwarf stars, we derived a slope of $0.31 \pm 0.06$ in the $[\mathrm{N} / \mathrm{Fe}]$ vs. $[\mathrm{Fe} / \mathrm{H}]$ plane for dwarfs with and without planets fitted together. This is much steeper than the one derived by Ecuvillon et al. (2004) for their sample of solar type stars with and without planets $(0.10 \pm 0.05)$ based on the NH band at $3360 \AA$. We checked whether our [N/Fe] correlates with the effective temperature or the surface gravity, and no significant trend is observed in either case. However, it seems that our dwarf stars with temperatures higher than the solar value either have $[\mathrm{N} / \mathrm{Fe}]$ close to zero or are slightly overabundant, whereas those cooler than the Sun are slightly underabundant (see Fig. 9, top left panel). By performing the same fit in the $[\mathrm{N} / \mathrm{Fe}]$ vs. $[\mathrm{Fe} / \mathrm{H}]$ plane, but only to stars with $T_{\text {eff }}<5800 \mathrm{~K}$, we derive a slope of $0.28 \pm 0.06$, a number that is less steep but is still a significantly high value.

The decreasing $[\mathrm{O} / \mathrm{Fe}]$ with increasing metallicity observed for our sample of dwarfs agrees with the trends derived by Delgado Mena et al. (2010) and by González Hernández et al. (2010, 2013b), and also with the recent results by Bertran de Lis et al. (2015) for the $\mathrm{O}$ abundances based on the $\mathrm{O} I \lambda 6158.17$ and [O I] $\lambda 6300.30$ atomic lines.

The distributions of $[\mathrm{X} / \mathrm{H}]$ are shifted toward higher abundances (see Fig. 13) owing to the metal-rich nature of stars hosting giant planets, as already known and discussed in the literature (see, e.g., Neves et al. 2009; Adibekyan et al. 2012b). The exceptions in the current study are oxygen and barium, whose $[\mathrm{X} / \mathrm{H}]$ abundances show no clear enrichment in giant planet hosts (see discussion in Sect. 6). In other words, most of these 
R. da Silva et al.: Abundances in dwarfs, subgiants, and giants with and without planets

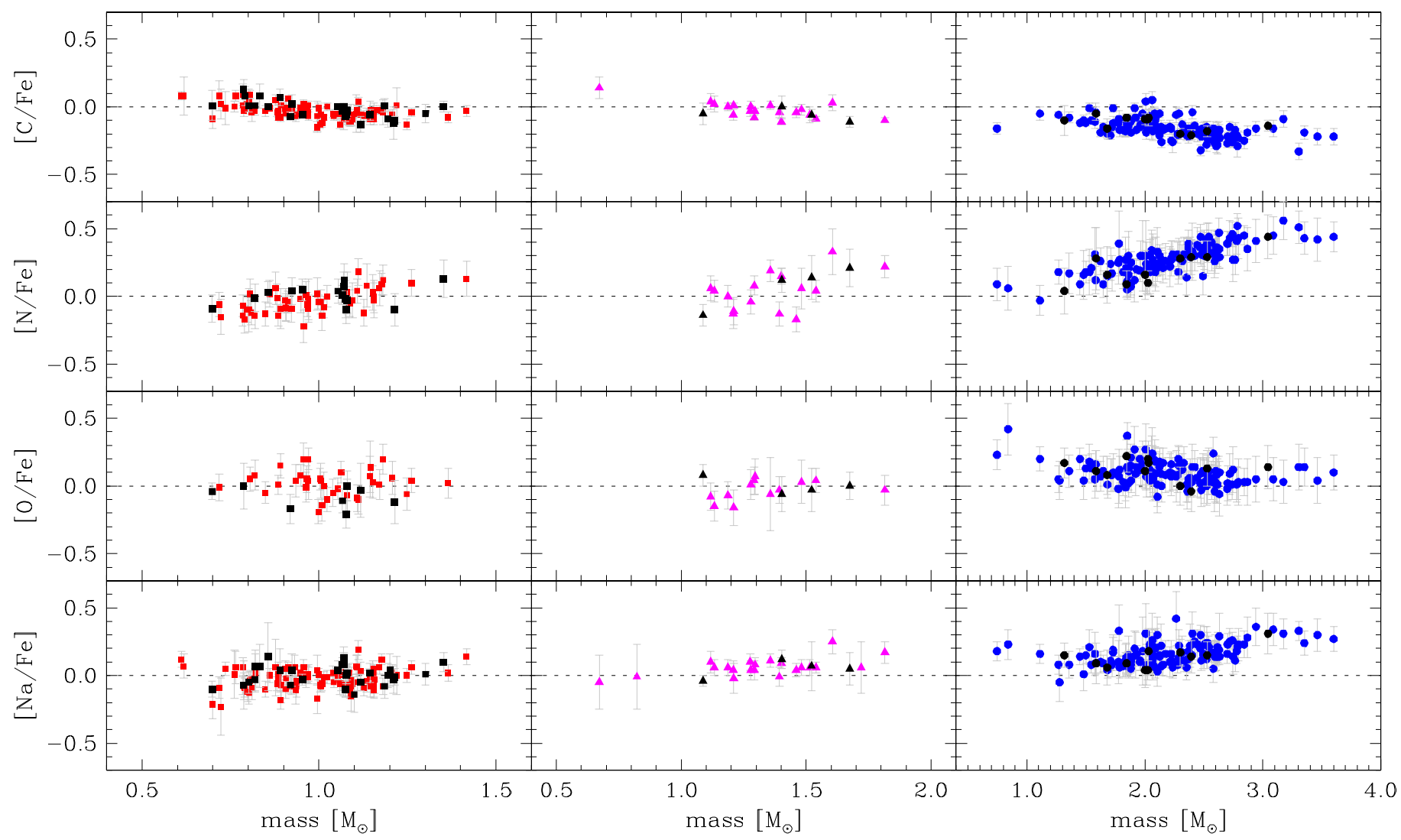

Fig. 8. Abundance ratios of $\mathrm{C}, \mathrm{N}, \mathrm{O}$, and $\mathrm{Na}$ as a function of mass for our sample of dwarfs (left panels), subgiants (middle panels), and giants (right panels). Stars with planets are represented by black symbols.

elements follow the same behavior of iron and are, for some reason, also linked to the planetary formation process. It is worth noting that only stars with giant planets (mass $\gtrsim 50$ Earth masses) were considered and compared with only stars for which no giant planet has been detected. We conducted an exhaustive search in the literature in order to classify each star and create these two subsamples. They are identified in the remarks column of Table 7, together with the respective reference. In addition, we note that we performed this comparison only for dwarf stars of the thin disk population. Evolved stars are normally not included in the surveys of planet search, because they are well known for their intrinsic radial-velocity variation due to pulsation and/or jitter (distortion in the observed signal caused by stellar magnetic activity). Subgiant stars could be added to the subsample of dwarfs, but we preferred to keep the homogeneity.

To quantify the observed trends with metallicity, we fitted linear regressions in the range of stars that are metal-poorer or metal-richer than the Sun, or in the whole range of metallicity, depending on which one provides a higher correlation. The abundance ratios were then subtracted by the predictions of these linear fits trying to remove (or at least minimize) the effects of the chemical evolution of the Galaxy's thin disk. The abundances corrected from such effects are used and discussed in Sect. 5.3, where we derive the abundance trends with the condensation temperature of the elements.

\subsubsection{Chemically peculiar stars}

Most of the dwarf and subgiant stars plotted in Figs. 5 and 6 follow the abundance trends with metallicity. A particular exception seems to be the vanadium abundance ratios for some of them. Such stars have $[\mathrm{V} / \mathrm{Fe}]$ higher than what is expected for their metallicity. By plotting the abundance ratios of the elements as a function of the other stellar parameters, we noticed that there is a systematic positive trend in $[\mathrm{V} / \mathrm{Fe}]$ as a function of the effective temperature for $T_{\mathrm{eff}} \gtrsim 6000 \mathrm{~K}$ for our sample of dwarfs (see Fig. 9, bottom left panel). For this reason, in the following sections we discuss the results obtained by including or excluding the vanadium abundances of stars with temperature higher than this limit. No clear trend in the abundance ratios as a function of temperature is observed for the other elements, or as a function of other stellar parameters (except age) either. This is the case at least for dwarfs and subgiants, because the elemental abundances in giants may have been affected by stellar evolution (see discussion in Sect. 5.1).

Concerning the chemical peculiarity of giant stars, two of our sample stars deserve special given their position in the $[\mathrm{Ba} / \mathrm{Fe}]$ vs. $[\mathrm{Fe} / \mathrm{H}]$ diagram of Fig. 7. Both HD 104979 and HD 202109 appear enriched in barium compared to other giants with the same metallicity. Williams (1971) derived a $[\mathrm{Ba} / \mathrm{Fe}]=1.1 \mathrm{dex}$ for HD 104979 and first suggested it to be a Ba II star ${ }^{2}$. More recent works have confirmed the barium overabundance for this star, namely $[\mathrm{Ba} / \mathrm{Fe}]=0.93 \pm 0.10 \mathrm{dex}$ (Zăcs 1994) and $[\mathrm{Ba} / \mathrm{Fe}]=0.54 \pm 0.31 \mathrm{dex}$ (Smiljanic et al. 2007), both in agreement with our determination of $[\mathrm{Ba} / \mathrm{Fe}]=0.77 \pm 0.06 \mathrm{dex}$.

2 A BaII star originates in the following scenario: when the more massive component of a binary system evolves as a thermally pulsing asymptotic giant branch star, the material produced in the He-burning envelope, enriched in s-process elements, is dredged up to the surface and then accreted by the companion by wind mass transfer. The initially more massive star finishes as a white dwarf, whereas the companion becomes a primary barium star. 


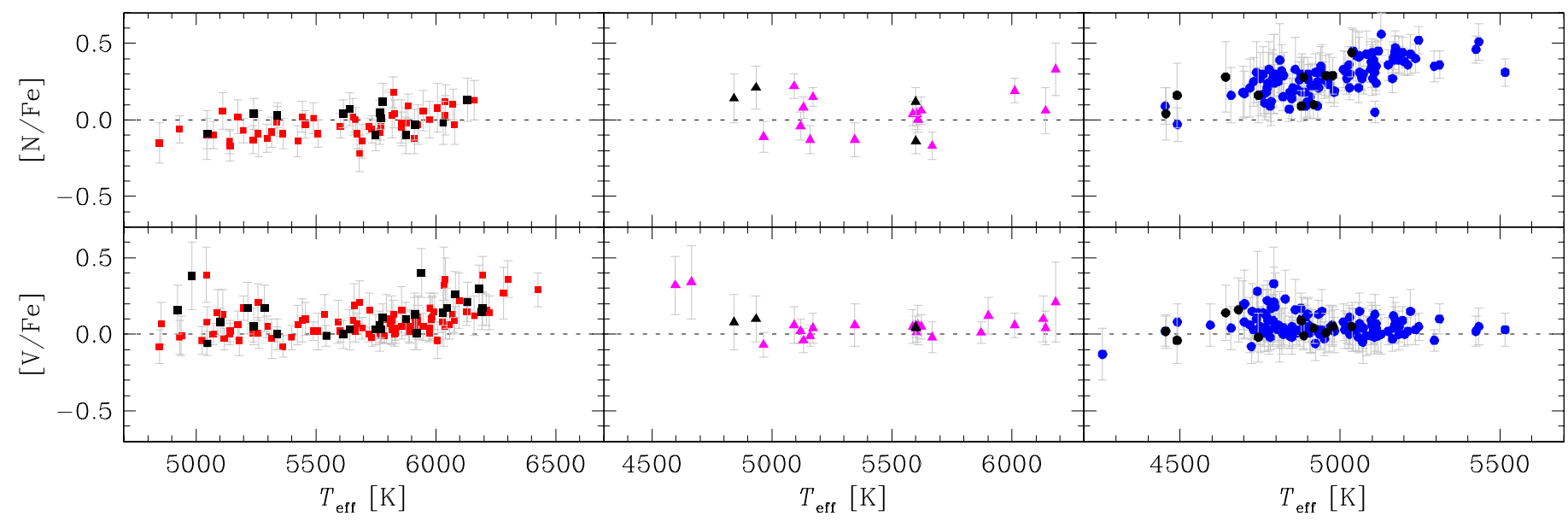

Fig. 9. $[\mathrm{N} / \mathrm{Fe}]$ and $[\mathrm{V} / \mathrm{Fe}]$ as a function of the effective temperature for our sample of dwarfs (left panels), subgiants (middle panels), and giants (right panels). Stars with planets are represented by black symbols.

The barium star nature of HD 202109 was first suggested by Chromey et al. (1969), who measured $[\mathrm{Ba} / \mathrm{Fe}]=0.8 \pm 0.3 \mathrm{dex}$, and was afterward classified as mild (marginal enriched) barium stars: $[\mathrm{Ba} / \mathrm{Fe}]=0.41 \pm 0.13$ dex by Zăcs $(1994)$ and $[\mathrm{Ba} / \mathrm{Fe}]=$ $0.31 \pm 0.26$ dex by Smiljanic et al. (2007). We derived $[\mathrm{Ba} / \mathrm{Fe}]=$ $0.40 \pm 0.09 \mathrm{dex}$, which is a result that agrees with the mild barium star classification.

\subsection{Abundance trends with age}

Figure 10 shows the $[\mathrm{X} / \mathrm{Fe}]$ abundance ratios as a function of age for the subsample of dwarf stars in the thin disk population. Similar to what is described in Sect. 5.1 in the case of the metallicity, we also quantified the observed trends with age by fitting linear regressions in the range of stars younger or older than the Sun, or in the whole range of age, depending on which one provides a higher correlation. Again, the abundance ratios were subtracted by the predictions of these linear fits in order to remove (or at least minimize) the effects of the chemical evolution of the Galaxy, using another approach,. The abundances corrected from such effects are used and discussed in Sect. 5.3, where we derive the trends with the condensation temperature of the elements.

Depending on the position of the star in the HR diagram, the errors in age may be quite high, and the age determination is thus less reliable. By means of the distribution of the uncertainties on this parameter, we set a $2 \sigma$ confidence limit up to which about $95 \%$ of the stars are found to have errors smaller than that limit, namely $\sim 4$ Gyr. Only stars with $\sigma($ age $)<4$ Gyr were therefore considered in the analysis.

\subsection{Abundance trends with condensation temperature}

During the past few years, several works have investigated the relation between the stellar chemical abundances and the condensation temperature $\left(T_{\mathrm{C}}\right)$ of the elements in stars with and without planets. Aiming to minimize systematic errors, the abundance difference between the Sun and other stars, $\Delta[\mathrm{X} / \mathrm{Fe}]$, has been used, where the solar abundances are derived from a Moon's spectrum, for example.

On the one hand, it has been suggested that the observed trends of $\Delta[\mathrm{X} / \mathrm{Fe}]$ as a function of $T_{\mathrm{C}}$ may have some relation with the planetary formation, in particular, with the formation of terrestrial planets (see the review performed by Ramirez et al. 2010). On the other hand, other authors argue that this kind of relation practically vanishes after removing the Galactic chemical evolution effects (see González Hernández et al. 2013b, and references therein). Indeed, positive slopes in these diagrams may be the result of having higher abundances of less refractory elements, such as $\mathrm{Na}$ and $\mathrm{Cu}$, with respect to more refractory ones, such as $\mathrm{Ti}$ and $\mathrm{Ca}$. In Fig. 5, both $[\mathrm{Ti} / \mathrm{Fe}]$ and $[\mathrm{Ca} / \mathrm{Fe}]$ have a slight decrease with increasing metallicity, whereas $[\mathrm{Na} / \mathrm{Fe}]$ and $[\mathrm{Cu} / \mathrm{Fe}]$ clearly increase for higher metallicities.

In particular, some of the studies discussed in the papers above consider only solar twins $\left(T_{\text {eff }}=5777 \pm 100 \mathrm{~K},[\mathrm{Fe} / \mathrm{H}]=\right.$ $0.0 \pm 0.1$ dex, $\log g=4.44 \pm 0.10 \mathrm{dex})$ or solar analogs $\left(T_{\mathrm{eff}}=\right.$ $5777 \pm 200 \mathrm{~K},[\mathrm{Fe} / \mathrm{H}]=0.0 \pm 0.2 \mathrm{dex}, \log g=4.44 \pm 0.20 \mathrm{dex})$ in their analysis. Recently, Adibekyan et al. (2014) have published the results of their study in which they searched for correlations of the $\Delta[\mathrm{X} / \mathrm{Fe}]$ vs. $T_{\mathrm{C}}$ trends with some stellar and orbital parameters. After correcting the abundances from the Galactic chemical evolution effects, they found that the observed trends correlate with age and anticorrelate with the surface gravity, and possibly with the mean Galactocentric distance, and that the correlations (or anticorrelations) are even steeper and more evident if only solar analogs are plotted.

Here we also investigate the relations involving $\Delta[\mathrm{X} / \mathrm{Fe}]$ as a function of the condensation temperature of the elements. To compute $[\mathrm{X} / \mathrm{Fe}]_{\text {Sun }}$, we used the solar abundances derived from the spectrum of the sunlight reflected by the Moon. First, we fit linear regressions for elements with $T_{\mathrm{C}}>900 \mathrm{~K}$, a limit normally used to distinguish refractory from volatiles. Values of $T_{\mathrm{C}}$ were taken from Lodders et al. (2009). To account for any effects that the Galactic chemical evolution may have on the observed trends, we applied the corrections based on both metallicity and age, as described previously. Figure 11 shows a few examples. Table 8, which is available in electronic form at the CDS, lists the slopes of the linear regressions and the respective standard deviations for thin disk dwarfs. Only dwarf stars were used in this analysis because their abundances are normally not affected by stellar evolution. The table contains the slopes and their uncertainties, the correlation coefficients, and the standard deviations of the regressions before and after applying the corrections for the Galactic chemical evolution effects based on age and on metallicity.

We then plot (Fig. 12) the respective slopes as a function of the stellar parameters $T_{\text {eff }},[\mathrm{Fe} / \mathrm{H}], \log g, \xi$, mass, and age. In this 
R. da Silva et al.: Abundances in dwarfs, subgiants, and giants with and without planets

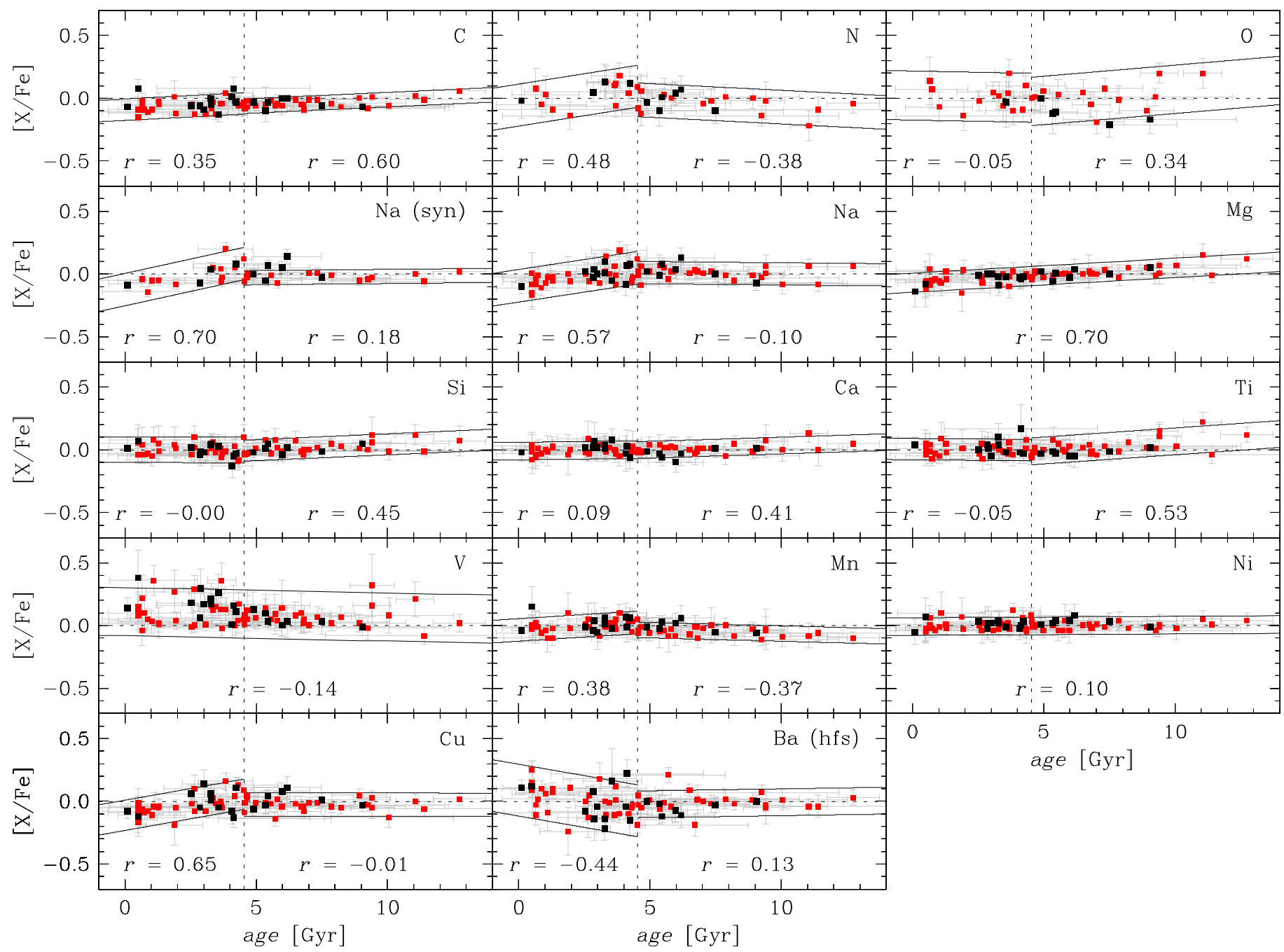

Fig. 10. Abundance ratios as a function of age for our subsample of 120 dwarf stars of the thin disk population. Stars with planets are indicated by black symbols. For sodium, the abundance ratios from both equivalent widths and spectral synthesis determination are plotted. The vertical dashed line indicates the adopted solar age $(4.53 \mathrm{Gyr})$. The $95 \%$ confidence intervals (solid lines) around linear regressions and their respective correlation coefficients $(r)$ are also shown. The linear regressions were derived either for age $\leq 4.53 \mathrm{Gyr}$, age $\geq 4.53 \mathrm{Gyr}$, or for the whole range in age, depending on which one has the more significant slope.

figure, solar analogs are distinguished from non-solar analogs and stars with planets from those without planets. Regarding the sample of solar analogs, the figure shows: i) a marginal correlation with metallicity (in the sense that metal-richer stars have more positive slopes) and with mass after removing the effects of chemical evolution based on age; however, these correlations completely disappear if the correction is based on metallicity; ii) a clear anticorrelation with the surface gravity in both scenarios of Galactic chemical evolution correction; iii) a marginal correlation with age, slightly steeper after applying the correction based on metallicity. The directions of these correlations agree with those reported by Adibekyan et al. (2014). We cannot check the $T_{\mathrm{C}}$ trends with Galactocentric distance because we only have solar neighborhood stars (distance to the Sun $<100 \mathrm{pc}$ ). Concerning the relation with the presence of planets, the small number of planet hosts in our sample of solar analogs prevents us from any conclusions.

By excluding the vanadium abundances of stars with $T_{\text {eff }} \gtrsim$ $6000 \mathrm{~K}$ from the analysis, the correlations and anticorrelations obtained above are still the same, specially because this limit is already imposed when only solar analogs are used.

\section{Statistical analysis}

We have used a number of statistical methods (see Breiman et al. 1984) to explore the multivariate properties of our sample. We are interested in uncovering significant differences in the stellar properties for stars with and without a known giant planet. Hereafter we use the indices 1 and 0 to distinguish these two samples, respectively. Moreover, the sample of dwarf stars is prefixed by the letter " $d$ ". Thus, samples $d 1$ and $d 0$ stand for dwarfs with and without a known giant planet, respectively. The analysis and the following discussion are restricted to the dwarfs because their sample is thought to be less affected by an observation bias.

\subsection{Abundance distributions}

We compare the abundance distributions between the samples d0 (red) and d1 (blue) in Fig. 13. The boxes and whiskers summarize percentiles of these abundance distributions graphically. Particularly, the middle line inside each box indicates the median for that distribution, whereas the upper and lower edges of 

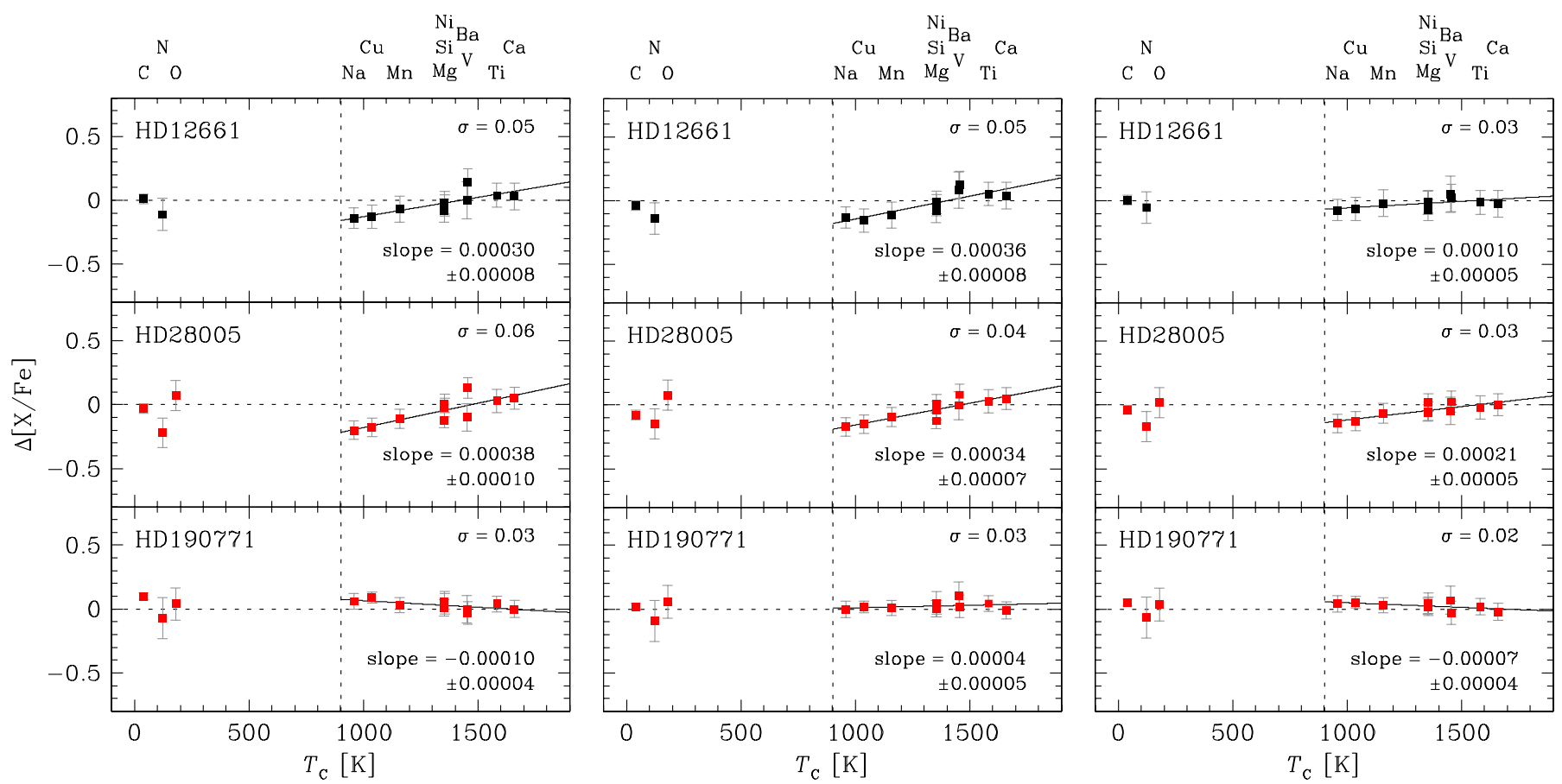

Fig. 11. Abundance differences $[\mathrm{X} / \mathrm{Fe}]_{\text {Sun }}-[\mathrm{X} / \mathrm{Fe}]_{\text {stars }}$ as a function of the condensation temperature of the elements for some of our dwarf stars of the thin-disk population. We show the trends before (left panels) and after applying the corrections for the Galactic chemical evolution effects based on age (middle panels) and on metallicity (right panels). The standard deviations of the linear regressions (solid lines) for $T_{\mathrm{C}}>900 \mathrm{~K}$ and their respective slopes are also shown. The planet host star is indicated by black symbols.

the box indicate the interquartile range. We applied a $2 \sigma$ clipping in the abundance data of each single element in order to get rid of outliers in the abundance space. Panel (a) shows the comparison for all dwarf stars after applying the sigma clipping, while panel (b) restricts the comparison to those stars having $-0.25 \leq[\mathrm{Fe} / \mathrm{H}]<+0.35$ (a range in common between the subsamples of stars with and without planets). Both comparisons depict that dwarf stars with giants planets (d1) have generally larger abundances in nearly all elements compared to dwarfs without giant planets $(\mathrm{d} 0)$. We note that vanadium seems to be exceptionally abundant in d1 stars, whereas no significant difference is seen between $\mathrm{d} 1$ and $\mathrm{d} 0$ for the oxygen and barium abundances. Concerning vanadium, the overabundance of $\mathrm{d} 1$ with respect to d0 does not change if only stars with $T_{\text {eff }} \lesssim 6000 \mathrm{~K}$ are used.

We used the Welsch's t-test to quantitatively assess whether the means of these distributions are significantly different from one another. Table 1 gives the test statistics for stars having $-0.25 \leq[\mathrm{Fe} / \mathrm{H}]<+0.35$, after applying $2 \sigma$ clipping. Some $p$-values are very low $(<0.001)$, reinforcing the visual comparison provided by Fig. 13. The mean of the abundance distribution of all elements, but $\mathrm{O}$ and $\mathrm{Ba}$, are higher in $\mathrm{d} 1$ stars, compared to d0 stars. Oxygen abundances are not represented very well in our sample, and only $32 \mathrm{~d} 0$ and $4 \mathrm{~d} 1$ stars have measured $\mathrm{O}$ abundance; this could be one of the reasons for the t-test giving a high $p$-value for the $\mathrm{O}$ abundance distribution comparison. Notwithstanding, it is highly interesting, although not clear, why $\mathrm{Ba}$ is not particularly more abundant in $\mathrm{d} 1$ stars.

The t-tests described above can only be applied to the abundance distribution of a single element at a time. They can pinpoint elements that are particularly more or less abundant in one of the tested distributions. To use the information encoded in the whole abundance space, we also applied a multivariate analysis
Table 1. Probabilities of the t-test statistics and coefficients of the linear discriminant for the $\mathrm{d} 0$ and $\mathrm{d} 1$ classes.

\begin{tabular}{ccccc}
\hline \hline $\begin{array}{c}\text { Abundance } \\
\text { ratio }\end{array}$ & $N(\mathrm{~d} 0)$ & $N(\mathrm{~d} 1)$ & $p$-value & $\begin{array}{c}\text { Linear } \\
\text { discriminant }\end{array}$ \\
\hline$[\mathrm{Fe} / \mathrm{H}]$ & 94 & 23 & $2.39 \times 10^{-4}$ & -0.87 \\
{$[\mathrm{C} / \mathrm{H}]$} & 86 & 21 & $5.12 \times 10^{-4}$ & $\ldots$ \\
{$[\mathrm{N} / \mathrm{H}]$} & 51 & 10 & $6.82 \times 10^{-3}$ & $\ldots$ \\
{$[\mathrm{O} / \mathrm{H}]$} & 36 & 5 & $9.21 \times 10^{-1}$ & $\ldots$ \\
{$[\mathrm{Na} / \mathrm{H}]$} & 48 & 10 & $4.92 \times 10^{-3}$ & $\ldots$ \\
{$[\mathrm{Mg} / \mathrm{H}]$} & 89 & 20 & $8.38 \times 10^{-4}$ & -5.43 \\
{$[\mathrm{Si} / \mathrm{H}]$} & 87 & 23 & $1.43 \times 10^{-4}$ & 0.70 \\
{$[\mathrm{Ca} / \mathrm{H}]$} & 88 & 20 & $7.62 \times 10^{-6}$ & -0.14 \\
{$[\mathrm{Ti} / \mathrm{V}]$} & 79 & 21 & $2.76 \times 10^{-4}$ & 8.23 \\
{$[\mathrm{~V} / \mathrm{H}]$} & 85 & 21 & $2.03 \times 10^{-9}$ & 2.27 \\
{$[\mathrm{Mn} / \mathrm{H}]$} & 91 & 23 & $3.56 \times 10^{-5}$ & -3.50 \\
{$[\mathrm{Ni} / \mathrm{H}]$} & 88 & 23 & $1.64 \times 10^{-4}$ & 5.62 \\
{$[\mathrm{Cu} / \mathrm{H}]$} & 81 & 22 & $6.76 \times 10^{-4}$ & 0.99 \\
{$[\mathrm{Ba} / \mathrm{H}]$} & 82 & 22 & $1.91 \times 10^{-1}$ & -2.01 \\
\hline
\end{tabular}

Notes. The second and third columns gives the number of stars in each group. The $p$-value gives the probability that the mean of both $\mathrm{d} 0$ and $\mathrm{d} 1$ distributions are statistically similar.

of variance (MANOVA) to assess the significance of differences in the multivariate means (that is, the location of samples 0 and 1 in abundance space). According to this MANOVA, we can reject the null hypothesis that the $\mathrm{d} 0$ and $\mathrm{d} 1$ samples have similar locations in abundance space $(p=0.006)$.

\subsection{Abundance difference per metallicity bin}

We have also considered a different approach to disentangling the effects of the chemical evolution of the Galaxy from the 
R. da Silva et al.: Abundances in dwarfs, subgiants, and giants with and without planets
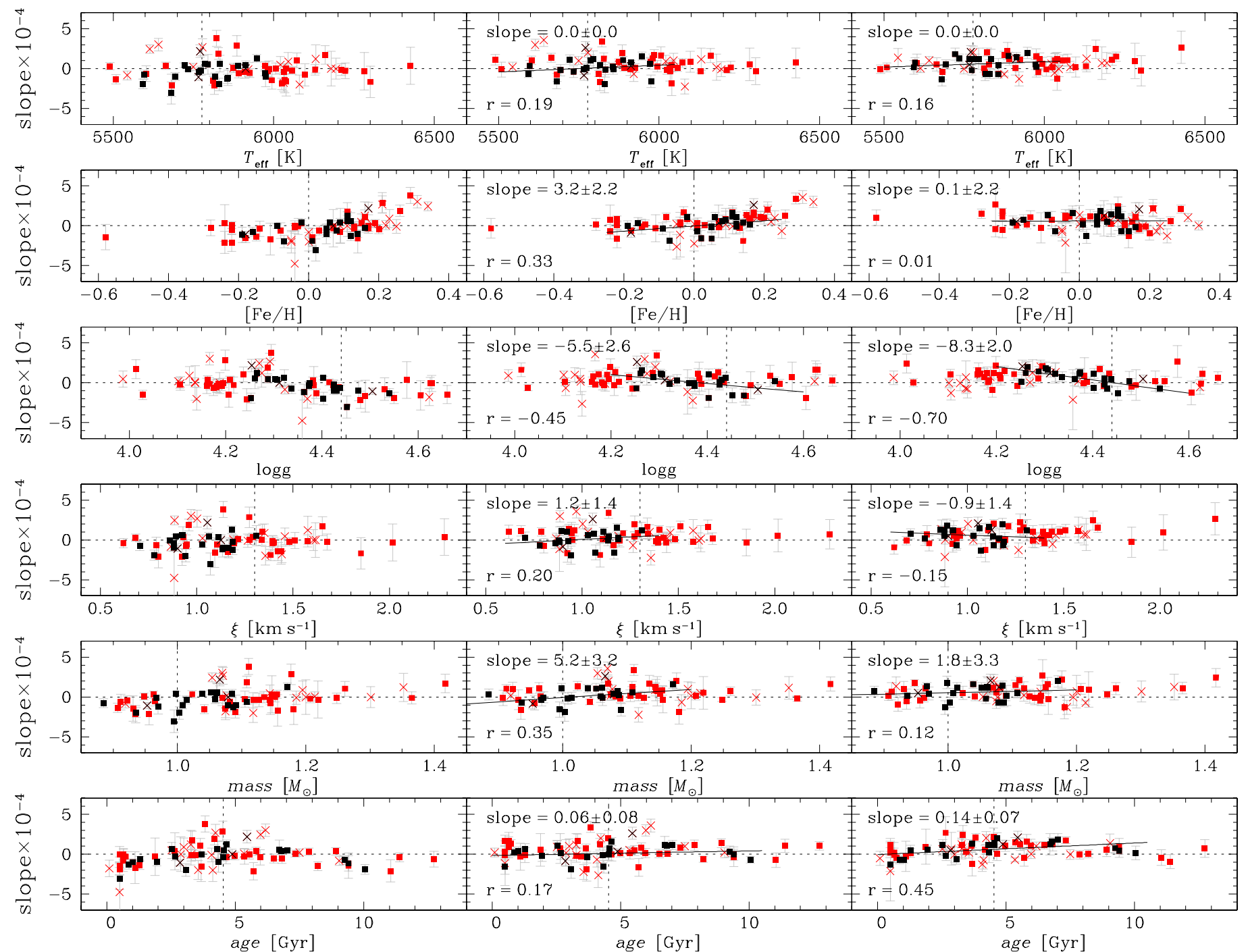

Fig. 12. Slope of the regressions computed in the diagrams $\Delta[\mathrm{X} / \mathrm{Fe}]$ vs. $T_{\mathrm{C}}$ (Fig. 11) plotted as a function of the derived stellar parameters. We show possible trends before (left panels) and after applying the corrections for the Galactic chemical evolution effects based on age (middle panels) and on metallicity (right panels). Dwarf stars of the thin disk population are separated in solar analogs (black symbols) and non-solar analogs (red symbols) Stars with planets are indicated by crosses. The slopes of linear regressions (solid lines) applied to the solar analogs and their respective correlation coefficients $(r)$ are also shown.

metallicity-giant planet connection. We defined a crude abundance quantity $\mathcal{Z}=[\mathrm{Mg} / \mathrm{H}]+[\mathrm{Si} / \mathrm{H}]+[\mathrm{Ca} / \mathrm{H}]+[\mathrm{Ti} / \mathrm{H}]+$ $[\mathrm{V} / \mathrm{H}]+[\mathrm{Mn} / \mathrm{H}]+[\mathrm{Ni} / \mathrm{H}]+[\mathrm{Cu} / \mathrm{H}]+[\mathrm{Ba} / \mathrm{H}]$. The symbol $\mathcal{Z}$ does not have an exact physical meaning, on account of the sum of unscaled log abundances, but it can serve as an enrichment index for the purpose of this analysis. For instance, it is clear that $\mathcal{Z}$ and $[\mathrm{Fe} / \mathrm{H}]$ should be correlated as a result of the Galactic chemical evolution. This index could only be defined for those stars having a complete set of abundances of the elements $\mathrm{Mg}$ to $\mathrm{Ba}$, including $\mathrm{Fe}$. $\mathrm{CNO}$ and $\mathrm{Na}$ abundances were not included in the $\mathcal{Z}$ definition because this would substantially lower the final number of stars having a complete set of abundance measurements.

The index was used in a linear model of the type

$\mathcal{Z}=\hat{\beta}_{0}+\hat{\beta}_{1}[\mathrm{Fe} / \mathrm{H}]+\epsilon$,

fit by least squares to the $\mathrm{d} 0$ stars. The residuals of the fit are given by $\epsilon$ for each star. Following this, we calculated the residuals of the $\mathrm{d} 1$ stars with respect to the same linear model fit to the d0 stars. A Welch's t-test applied to these residual distributions indicates that the difference in their means are significant (a $p$-value $p=0.0023$ ), with $\bar{\epsilon}_{\mathrm{d} 1}-\bar{\epsilon}_{\mathrm{d} 0}=0.16 \pm 0.10$. That is, for a given $[\mathrm{Fe} / \mathrm{H}], \mathrm{d} 1$ stars have generally larger $\mathcal{Z}$ indices than do d0 stars. If we can consider the d0 stars as a "null hypothesis" population, for which the elemental abundances only reflect the Galactic chemical evolution, the residuals of $\mathcal{Z}$ for the $\mathrm{d} 1$ stars using the linear model in Eq. (1) should have no chemical evolution bias. Again, if we calculate $\mathcal{Z}$ excluding $[\mathrm{V} / \mathrm{H}]$, this result practically does not change (the new $p$-value would be $p=0.0026$ ). On the other hand, if $\mathcal{Z}$ is calculated excluding the $[\mathrm{Ba} / \mathrm{H}]$ abundance ratios, we obtain $p=0.0005$ and $\bar{\epsilon}_{\mathrm{d} 1}-\bar{\epsilon}_{\mathrm{d} 0}=0.22 \pm 0.12$; i.e., the difference between the $\mathrm{d} 0$ and $\mathrm{d} 1$ populations is even greater.

We defined $\mathcal{Z}$ by mixing elements with different nucleosynthetic origins, which might veil the real difference in abundance between stars with and without planets. However, the mean $[\mathrm{X} / \mathrm{H}]$ distributions for $\alpha(\mathrm{Mg}, \mathrm{Si}, \mathrm{Ca}, \mathrm{Ti})$ and for iron-peak $(\mathrm{V}$, $\mathrm{Cr}, \mathrm{Mn}, \mathrm{Co}, \mathrm{Ni}$ ) elements in stars hosting giant planets are very similar, as reported by Mata Sánchez et al. (2014). Indeed, if 

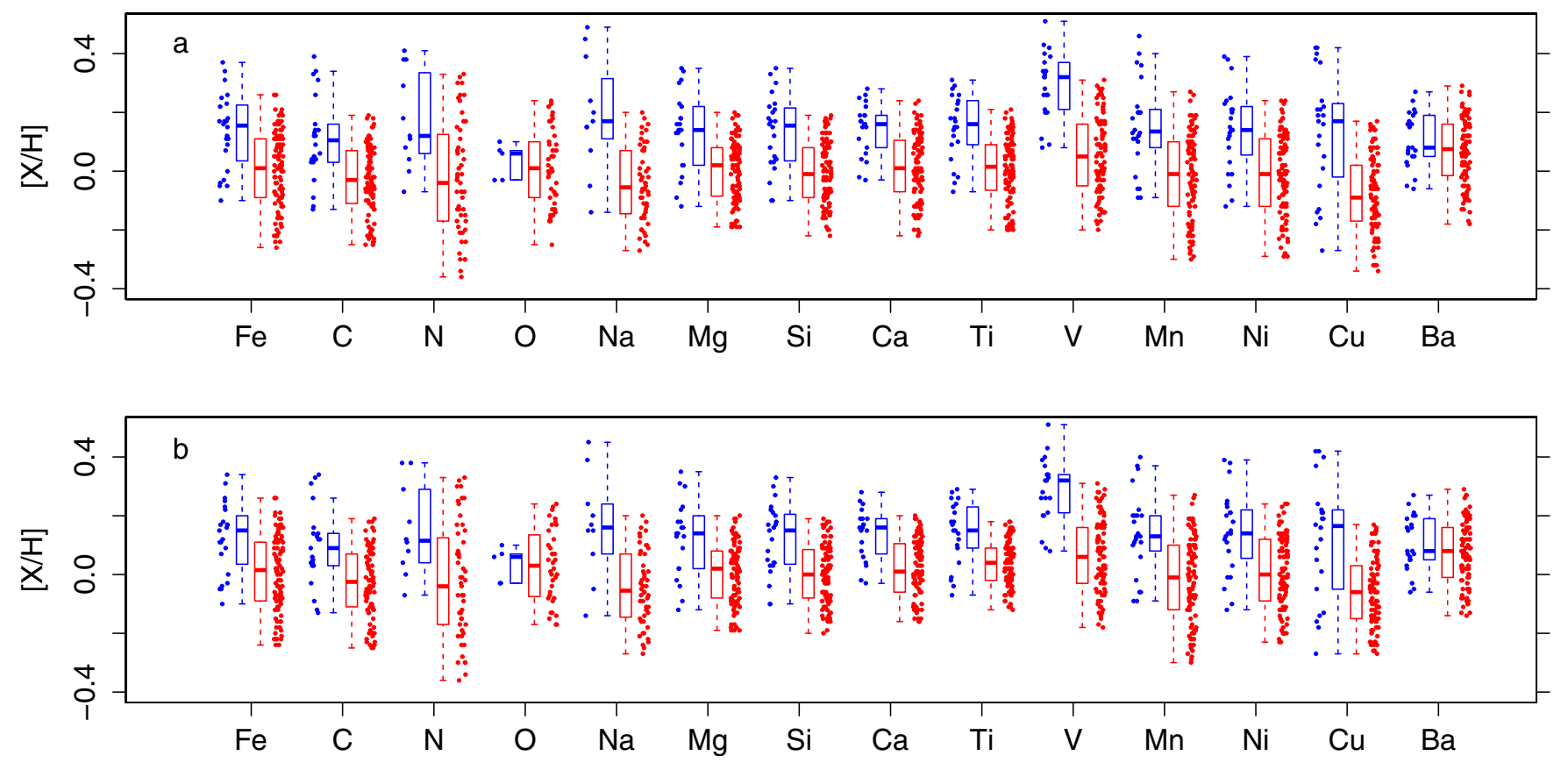

Fig. 13. Boxplot for the comparison between the elemental abundances of dwarf stars with and without planets, shown in blue and red, respectively. Two-sigma clipping was applied to all abundance distributions before the comparison, to avoid abundance outliers. The top panel shows the distributions for dwarfs in the whole $[\mathrm{Fe} / \mathrm{H}]$ abundance space, whereas the bottom panel restricts the comparison to those stars having $-0.25 \leq$ $[\mathrm{Fe} / \mathrm{H}]<+0.35$. The original data points are shown beside each corresponding box to allow visualization of the intrinsic scatter.

Table 2. Excerpt from the list of atomic line parameters of the elements used in the analysis and $E W$ s measured in the degraded spectrum of the Solar Flux Atlas.

\begin{tabular}{ccccc}
\hline \hline$\lambda[\AA]$ & Id. & $\begin{array}{c}\chi \\
{[\mathrm{eV}]}\end{array}$ & $\log g f$ & $\begin{array}{c}E W \\
{[\mathrm{~m} \AA]}\end{array}$ \\
\hline 5247.06 & $\mathrm{Fe}_{\mathrm{I}}$ & 0.09 & -5.022 & 64.0 \\
5322.05 & $\mathrm{Fe}_{\mathrm{I}}$ & 2.28 & -2.878 & 62.6 \\
5501.48 & $\mathrm{Fe}_{\mathrm{I}}$ & 0.96 & -3.105 & 121.2 \\
5522.45 & $\mathrm{Fe}_{\mathrm{I}}$ & 4.21 & -1.397 & 46.1 \\
5543.94 & $\mathrm{Fe}_{\mathrm{I}}$ & 4.22 & -1.055 & 64.7 \\
5546.51 & $\mathrm{Fe}$ I & 4.37 & -1.098 & 54.6 \\
5560.22 & $\mathrm{Fe}$ I & 4.43 & -1.080 & 52.8 \\
5587.58 & $\mathrm{Fe}_{\mathrm{I}}$ & 4.14 & -1.738 & 32.0 \\
5618.64 & $\mathrm{Fe}_{\mathrm{I}}$ & 4.21 & -1.310 & 50.9 \\
5619.60 & $\mathrm{Fe}_{\mathrm{I}}$ & 4.39 & -1.422 & 36.2 \\
$\ldots$ & $\ldots$ & $\ldots$ & $\ldots$ & $\ldots$ \\
\hline
\end{tabular}

Notes. Lines with missing $g f$ values represent the elements for which the hyperfine structure was taken into account $(\mathrm{Mg}, \mathrm{V}, \mathrm{Mn}$, and $\mathrm{Cu})$ and the detailed line splitting is shown in Table 3 . The whole table is available in electronic form at the CDS.

we calculate $\mathcal{Z}$ for $\alpha$ elements alone, we obtain $\bar{\epsilon}_{\mathrm{d} 1}-\bar{\epsilon}_{\mathrm{d} 0}=$ $0.08 \pm 0.06$, whereas for only our iron-peak elements $(\mathrm{V}, \mathrm{Mn}$, $\mathrm{Ni}$ ), we have $\bar{\epsilon}_{\mathrm{d} 1}-\bar{\epsilon}_{\mathrm{d} 0}=0.10 \pm 0.06$. For stars without planets, the $[\mathrm{X} / \mathrm{H}]$ distributions are also very similar, having a maximum abundance at $\sim-0.1$ for most species (Mata Sánchez et al. 2014). Therefore, we conclude that the mix of elements when calculating $\mathcal{Z}$ is not a bad approach, especially because the differences that we are comparing become more significant when the abundances of all these elements are added together.

\subsection{Classification trees}

Assuming the general connection between chemical abundance and the presence of a giant planet, we can extend the reasoning
Table 3. Excerpt from the list of $g f$ values for lines with significant hyperfine structure computed based on the degraded spectrum of the Solar Flux Atlas.

\begin{tabular}{lccc}
\hline \hline Id. & $\lambda_{0}[\AA]$ & $\lambda[\AA]$ & $\log g f$ \\
\hline Mg I & 4571.10 & 4571.078 & -6.554 \\
Mg I & 4571.10 & 4571.087 & -6.594 \\
Mg I 4571.10 & 4571.096 & -5.694 \\
Mg I & 4730.04 & 4730.031 & -3.254 \\
Mg I & 4730.04 & 4730.038 & -3.294 \\
Mg I & 4730.04 & 4730.046 & -2.394 \\
Mg I 5711.10 & 5711.074 & -2.716 \\
Mg I 5711.10 & 5711.083 & -2.756 \\
Mg I $^{5}$ & 5711.10 & 5711.091 & -1.856 \\
$\ldots$ & $\ldots$ & $\ldots$ & $\ldots$ \\
\hline
\end{tabular}

Notes. $\lambda_{0}$ is the central wavelength of the spectral line, whereas $\lambda$ is the wavelength of the hyperfine component. The whole table is available in electronic form at the CDS.

in using the iron abundance as an indicator of the plausibility that the star harbors a planet. To do this, we applied a classification tree to a complete set of $[\mathrm{X} / \mathrm{H}]$ abundances for the dwarfs in our sample. Classification trees give the optimal splitting of observations coming from different classes (or populations). In our problem, we consider two classes: stars with and without giant planets. The tree is built through recursive partitioning of the abundance space into nested groups, aiming at terminal groups having the highest purity (that is, having most, if not all, of its members coming from a single class). Each classification tree has nodes, branches, and terminal leaves (or group). The nodes are annotated with some property (or variable) and a splitting rule that points to one branch or the other. The leaves are usually labeled with the statistics of the partitioned group. 
full partition model

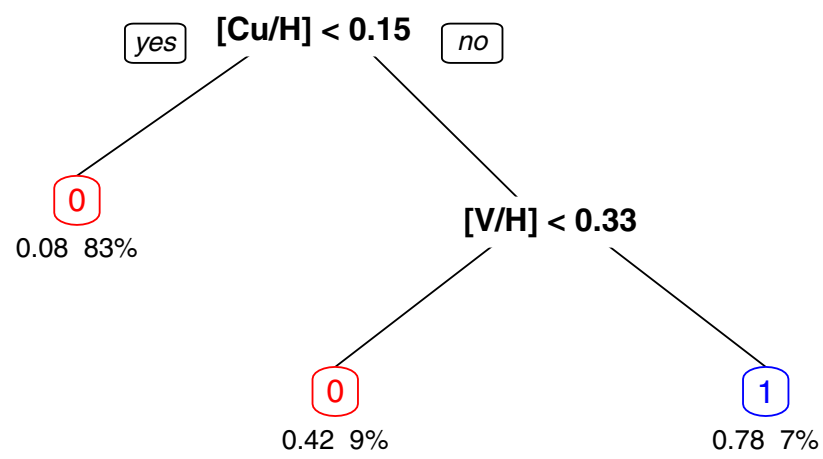

without Cu

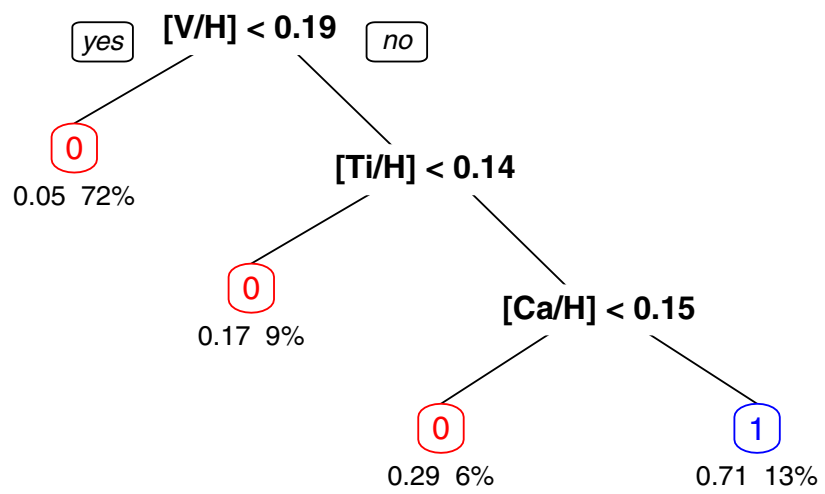

without V

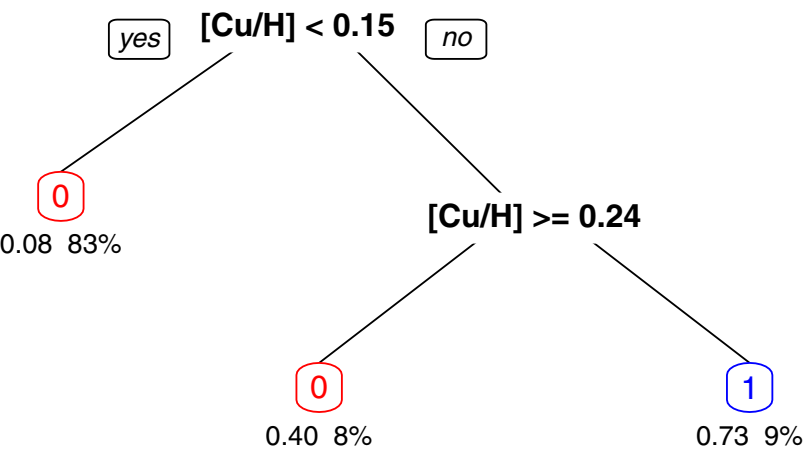

without V \& Cu

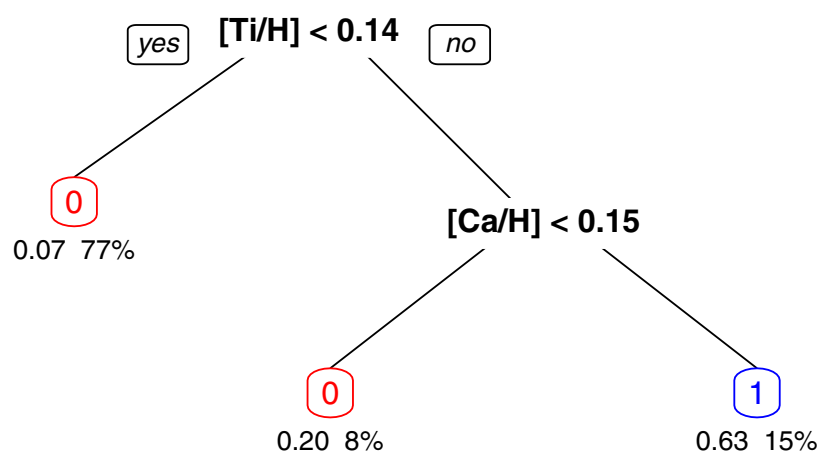

Fig. 14. Classification trees for the planet-harboring status among dwarf stars in our sample. The nodes are annotated with a categorical binary variable that assumes values 1 or 0 for stars with and without a giant planet, respectively. The leaves are marked with statistics of the partitioned group: fraction of $\mathrm{d} 1$ stars in the group (stars harboring a giant planets) and percentage of all dwarfs stars that were classified in that leaf. The top left tree is the full partition model for our sample. The other trees consider the case when two important elements are not known: $\mathrm{V}$ and $\mathrm{Cu}$, one at a time or both.

As previously explained, on account of the requirement of having a complete set of abundances, $\mathrm{CNO}$ and Na measurements were not used in this analysis. No sigma clipping to the abundances was used before applying the classification trees. We show in Fig. 14 four classification trees. The leaves are marked (and color-coded, for easy identification) with the most likely class of its members: 0 (red) if the star is not likely to harbor a giant planet, and 1 (blue) if the opposite holds. Two numbers are annotated below each terminal leaf, showing the fraction of d1 stars in the group (stars harboring a giant planet) and the percentage of all dwarfs stars that were classified in that leaf. Thus, the numbers $0.429 \%$ can be read as $9 \%$ of all dwarfs that were classified in that group and $42 \%$ of them that harbor a giant planet. The upper lefthand panel gives the classification tree when we consider all elements from $\mathrm{Mg}$ to $\mathrm{Ba}$. Although this abundance space is formed by the measurements of ten chemical species, only two of them are needed for an optimal partition: $\mathrm{Cu}$ and V. Copper abundances seem to be the best single indicator of the presence of a giant planet: only $8 \%$ of the stars having $[\mathrm{Cu} / \mathrm{H}]<0.15$ dex harbor a giant planet. Vanadium comes second in refining this: $78 \%$ of stars having $[\mathrm{Cu} / \mathrm{H}] \geq 0.15$ dex and $[\mathrm{V} / \mathrm{H}] \geq 0.33$ dex harbors a giant planet. The three other classification trees in Fig. 14 show the partition model when $\mathrm{Cu}$, $\mathrm{V}$, or both variables are not used. If vanadium is taken out of the independent variable set, the whole ten-dimensional abundance space could still be partitioned using the $\mathrm{Cu}$ abundances alone. The purity is somewhat lower: $73 \%$ of the stars having $0.15 \leq[\mathrm{Cu} / \mathrm{H}] \leq 0.24$ harbor a giant planet. If, on the other hand, we take $\mathrm{Cu}$ from the set of independent variables, $\mathrm{V}$ takes the lead as the most relevant element, followed by two more splittings using $\mathrm{Ti}$ and $\mathrm{Ca}$. The two more meaningful (extreme) leaves give: i) $5 \%$ of stars having $[\mathrm{V} / \mathrm{H}]<0.19$ dex harbor a giant planet; and ii) $71 \%$ of stars having simultaneously $[\mathrm{V} / \mathrm{H}] \geq 0.19$, $[\mathrm{Ti} / \mathrm{H}] \geq 0.14$ and $[\mathrm{Ca} / \mathrm{H}] \geq 0.15$ dex harbor a giant planet. Taking both $\mathrm{Cu}$ and $\mathrm{V}$ out of the set of independent variables yields a classification tree based on $\mathrm{Ti}$ and $\mathrm{Ca}$ abundances that is an exact subtree from the third case (with no $\mathrm{Cu}$ abundances).

\subsection{Linear discriminant analysis}

A similar, but more sophisticated, approach can be taken using a linear discriminant analysis (LDA), which finds the linear combination of observed properties (in our case, abundances) which best separates two or more classes of objects. The result is an index, or system of indices, that may act as a linear classifier. The difference from the classification tree is that this one uses a hierarchical recursive splitting of single properties, which 


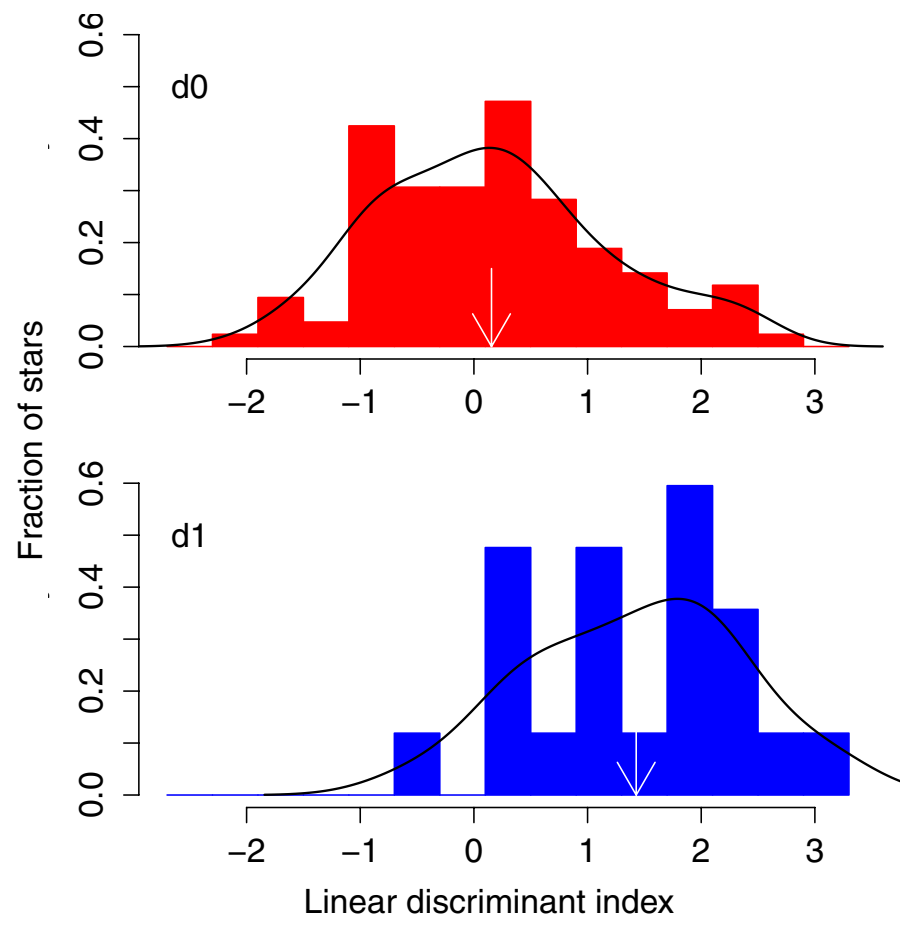

Fig. 15. Histograms of the linear discriminant indices defined in Table 1. The color code with blue and red is used to indicate stars with (top panel) and without planets (bottom panel), respectively. A black line shows the kernel density function for these indices, and a white arrow points to the position of the mean in each distribution.

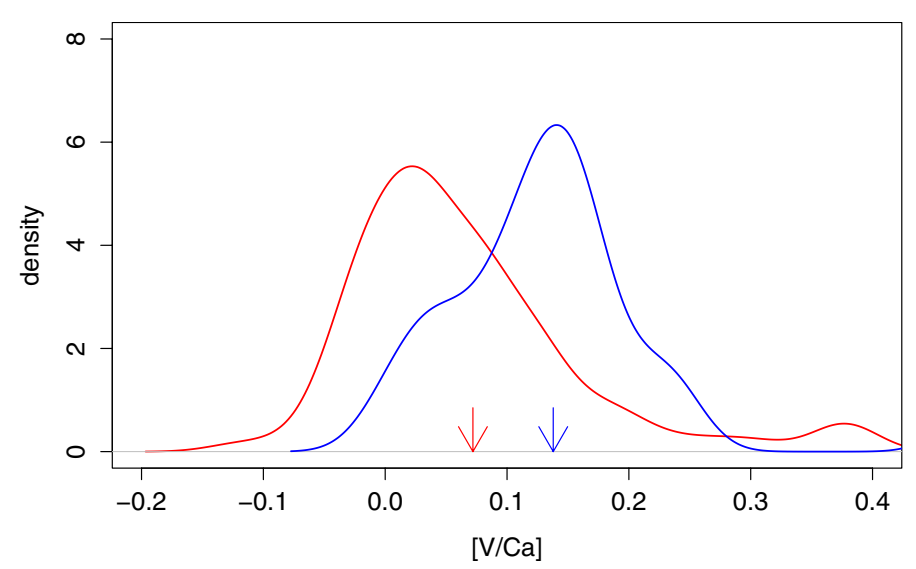

Fig. 16. Kernel probability density functions for the relevant abundance ratio, $[\mathrm{V} / \mathrm{Ca}]$, that allows the discrimination of dwarfs with and without giant planets. The color code blue and red is used to indicate stars with and without planets, respectively. These abundance ratio was found to distinguish dwarfs with respect to their planet-harboring status, in a similar role to what is played by the linear discriminant indices.

aims at classes with the highest purity possible, while the LDA searches for rotations of the original multiparametric space that leads to the best categorization of the classes in the entire space.

We applied the LDA to the complete abundance set of $\mathrm{Mg}$ to $\mathrm{Ba}$ for dwarfs, looking for the linear combination of abundances that could differentiate the $\mathrm{d} 0$ from $\mathrm{d} 1$ samples better. The resulting linear discriminant is given in the last column of Table 1 and in Fig. 15. As explained, the LDA finds the best linear combination of the original abundances that discriminates among the tested classes. The numbers in the table are the coefficients of this linear discriminant. An index formed by $-0.87[\mathrm{Fe} / \mathrm{H}]-5.43[\mathrm{Mg} / \mathrm{H}]+\ldots+0.99[\mathrm{Cu} / \mathrm{H}]-2.01[\mathrm{Ba} / \mathrm{H}]$ can thus be used to differentiate d0 from d1 stars. If our sample can be taken as unbiased and representative of the stellar abundances of stars with and without giant planets, we can use this discriminant index in a predictive way, when the giant planet-harboring status of the star is unknown a priori. As pointed out before, the concept is similar to using a classification tree, although now we use all abundances to build a single optimizer classifier. The power of this classifier can be seen in Fig. 15, where we show the histogram of the discriminant index.

We explored all possible combinations of abundance ratios involving the elements from $\mathrm{Mg}$ to $\mathrm{Ba}$, using the Welch test to assess interesting pairs. After considering all possibilities, we have found that $[\mathrm{V} / \mathrm{Ca}]$ seems to be a good abundance ratio for distinguishing dwarfs with planets: the average difference in $[\mathrm{V} / \mathrm{Ca}]$ between the $\mathrm{d} 0$ and $\mathrm{d} 1$ groups amounts to $0.07 \mathrm{dex}$, leading to the rejection of the null hypothesis with $p=0.005$.

Kernel probability density functions for the relevant abundance ratio that allows distinguishing dwarfs with and without giant planets are shown in Fig. 16. The color code is similar to the one used before: blue and red for stars with and without planets, respectively. The figure shows the $[\mathrm{V} / \mathrm{Ca}]$ among dwarf stars. Although there is considerable intersection between the red and blue curves, it is both remarkable and puzzling that this abundance ratio seems to distinguish stars according to their planet-harboring status.

\section{Conclusions}

Including more stars, with and without detected planets, and more elements in the current study aimed to improve the work presented in Paper I. A summary of our new results and conclusions follows:

i) The systematic differences in the abundance ratios of $\mathrm{C}, \mathrm{N}$, and $\mathrm{Na}$ among dwarfs, subgiants, and giants seem to confirm that mixing processes, together with cycles of nucleosynthetic reactions, do modify the abundances in the surface of evolved stars.

ii) Such mixing processes indeed seem to alter the $\mathrm{Na}$ photospheric abundances in giant stars, considering that, even when taking the influence of $\mathrm{C}_{2}$ and $\mathrm{CN}$ molecular features into account, a $\mathrm{Na}$ overabundance is still observed.

iii) The slopes in the $\Delta[\mathrm{X} / \mathrm{Fe}]$ vs. $T_{\mathrm{C}}$ diagrams show a correlation with age and an anticorrelation with the surface gravity, even after accounting for the effects of the Galactic chemical evolution. These results appear only when the sample is restricted to solar-analog stars; in other words, as also pointed out by Adibekyan et al. (2014), older and more evolved stars are in same way connected to a lower number of refractory elements available for the star formation. No significant correlation with other stellar parameters and no relation with the presence of planets are observed.

iv) Our statistical analysis has provided the following results.

a) The overabundance of other elements in dwarf stars hosting giant planets confirms the previous statement that not only is iron linked to the planetary formation process, but also C, N, Na, Mg, Si, Ca, Ti, V, Mn, Ni, and $\mathrm{Cu}$.

b) For $\mathrm{O}$ and $\mathrm{Ba}$ there are no significant differences between the samples of dwarfs with and without giant planets. oxygen is not very well represented in our analysis, but the result for barium is intriguing and deserves further investigation.

c) Vanadium is the element with the most significant abundance difference between giant planet hosts and single 
R. da Silva et al.: Abundances in dwarfs, subgiants, and giants with and without planets

Table 4. Excerpt from the list of 140 dwarf stars with the photospheric parameters and [Ti/Fe] abundance ratios.

\begin{tabular}{lcccccrr}
\hline \hline Star & $\begin{array}{c}\text { Spectral } \\
\text { type }\end{array}$ & $\begin{array}{c}V_{\text {broad }} \\
{\left[\mathrm{km} \mathrm{s}^{-1}\right]}\end{array}$ & $\begin{array}{c}T_{\text {eff }} \pm \sigma \\
{[\mathrm{K}]}\end{array}$ & $\log g \pm \sigma$ & $\begin{array}{c}\xi \pm \sigma \\
{\left[\mathrm{km} \mathrm{s}^{-1}\right]}\end{array}$ & \multicolumn{1}{c}{$[\mathrm{Fe} / \mathrm{H}] \pm \sigma$} & {$[\mathrm{Ti} / \mathrm{Fe}] \pm \sigma$} \\
\hline BD +290503 & G5 & 3.58 & $5180 \pm 50$ & $4.26 \pm 0.20$ & $0.86 \pm 0.31$ & $0.11 \pm 0.07$ & $-0.07 \pm 0.10$ \\
HD 10145 & G5 V & 0.00 & $5593 \pm 43$ & $4.25 \pm 0.19$ & $0.78 \pm 0.14$ & $0.01 \pm 0.07$ & $0.03 \pm 0.07$ \\
HD 10307 & G1.5 V & 2.72 & $5913 \pm 49$ & $4.29 \pm 0.22$ & $1.06 \pm 0.09$ & $0.09 \pm 0.06$ & $-0.02 \pm 0.07$ \\
HD 10476 & K1 V & 0.00 & $5141 \pm 31$ & $4.29 \pm 0.18$ & $0.40 \pm 0.25$ & $-0.05 \pm 0.05$ & $0.02 \pm 0.08$ \\
HD 106116 & G4 V & 0.00 & $5620 \pm 36$ & $4.22 \pm 0.17$ & $0.84 \pm 0.11$ & $0.16 \pm 0.05$ & $-0.01 \pm 0.09$ \\
HD 106516 & F9V & 7.26 & $6401 \pm 158$ & $4.85 \pm 0.39$ & $1.59 \pm 0.66$ & $-0.47 \pm 0.12$ & $0.29 \pm 0.17$ \\
HD 10780 & K0 V & 0.50 & $5339 \pm 26$ & $4.40 \pm 0.15$ & $0.71 \pm 0.13$ & $0.00 \pm 0.04$ & $0.02 \pm 0.06$ \\
HD 108954 & F9V & 3.43 & $6194 \pm 60$ & $4.60 \pm 0.23$ & $1.15 \pm 0.13$ & $0.08 \pm 0.08$ & $0.03 \pm 0.09$ \\
HD 109358 & G0V & 2.77 & $5875 \pm 30$ & $4.42 \pm 0.14$ & $1.21 \pm 0.08$ & $-0.22 \pm 0.04$ & $0.03 \pm 0.06$ \\
HD 11007 & F8 V & 3.40 & $6032 \pm 44$ & $4.16 \pm 0.26$ & $1.39 \pm 0.09$ & $-0.14 \pm 0.06$ & $0.03 \pm 0.09$ \\
$\ldots$ & $\ldots$ & $\ldots$ & $\ldots$ & $\ldots$ & $\ldots$ & $\ldots$ & $\ldots$ \\
\hline
\end{tabular}

Notes. The broadening velocity $V_{\text {broad }}$ is also shown. The whole tables for this and other elements, also for subgiants and giants, are available in electronic form at the CDS.

Table 7. Same as Table 4 but showing photometric and evolutionary parameters with the population membership.

\begin{tabular}{|c|c|c|c|c|c|c|c|c|c|c|}
\hline Star & $B-V$ & $M_{\mathrm{V}}$ & $\begin{array}{l}\text { Dist. } \\
\text { [pc] }\end{array}$ & $B C$ & $\log L$ & $\begin{array}{l}\text { Mass } \\
{\left[M_{\odot}\right]}\end{array}$ & $\begin{array}{l}\text { Age } \\
{[\mathrm{Gyr}]}\end{array}$ & $\begin{array}{l}\text { Population } \\
\text { group }\end{array}$ & Remarks & Ref. \\
\hline HD 10145 & 0.691 & 4.84 & 37.3 & -0.106 & $0.005 \pm 0.026$ & $0.94 \pm 0.03$ & $10.1^{+2.5}-1.8$ & thin & & \\
\hline HD 10476 & 0.836 & 5.82 & 7.5 & -0.239 & $-0.331 \pm 0.006$ & $0.78 \pm 0.04$ & $17.1^{+8.2}-7.7$ & thin & $a$ & \\
\hline HD 106116 & 0.701 & 4.79 & 34.9 & -0.100 & $0.025 \pm 0.015$ & $1.00 \pm 0.03$ & $7.1^{+2.5}-1.4$ & thin/thick & & \\
\hline HD 106516 & 0.470 & 4.36 & 22.4 & 0.011 & $0.150 \pm 0.016$ & $1.05 \pm 0.05$ & $0.2^{+1.3}-0.2$ & thick & & \\
\hline HD 108954 & 0.568 & 4.53 & 21.8 & -0.010 & $0.092 \pm 0.008$ & - & - & thin & $b$ & \\
\hline HD 124292 & 0.733 & 6.18 & 22.1 & -0.143 & $-0.516 \pm 0.011$ & - & - & thin & $e$ & 3 \\
\hline HD 128311 & 0.973 & 6.38 & 16.6 & -0.126 & $-0.603 \pm 0.015$ & - & 0.50 & thin & ${ }^{c, d}(\mathrm{U})$ & 1,2 \\
\hline HD 206860 & 0.587 & 4.74 & 17.9 & -0.020 & $0.017 \pm 0.008$ & - & 0.10 & thin & ${ }^{c, d}(\mathrm{P})$ & 1,2 \\
\hline HD 25825 & 0.593 & 4.50 & 45.9 & -0.021 & $0.108 \pm 0.052$ & - & 0.65 & thin & ${ }^{d}(\mathrm{H})$ & 4 \\
\hline HD 42807 & 0.663 & 5.17 & 18.0 & -0.070 & $-0.140 \pm 0.008$ & - & 2.70 & thin & ${ }^{d}(\mathrm{~W})$ & 5 \\
\hline$\ldots$ & $\ldots$ & $\ldots$ & $\ldots$ & $\ldots$ & $\ldots$ & $\ldots$ & $\ldots$ & $\ldots$ & & \\
\hline
\end{tabular}

Notes. ${ }^{(a)}$ Not considered in the abundance analysis because the age uncertainties are larger than 4 Gyr (see Sect. 4.1 ); ${ }^{(b)}$ mass and age not derived because the star is located outside the evolutionary tracks and isochrones used; ${ }^{(c)}$ star hosting at least one giant planet; ${ }^{(d)}$ member of a stellar moving group (U: Ursa Major; P: Pleiades; H: Hyades; W: Wolf 630); ${ }^{(e)}$ subdwarf candidate.

References. (1) http: //exoplanet. eu; (2) Montes et al. (2001); (3) Sandage \& Fouts (1986); (4) Eggen (1950); (5) Bubar \& King (2010).

dwarfs, and this difference is still significant if only stars with $T_{\text {eff }} \lesssim 6000 \mathrm{~K}$ are used.

d) By combining the elements from $\mathrm{Mg}$ to $\mathrm{Cu}$ according to Eq. (1), we found a significant overabundance, for the same metallicity, in giant planet hosts in comparison with our sample of single dwarfs.

e) Based on the classification tree analysis we have found that abundances of copper and calcium are special discriminators of dwarfs with and without giant planets (over the $[\mathrm{X} / \mathrm{H}]$ scale).

f) By means of a linear discriminant analysis, we have found that $[\mathrm{V} / \mathrm{Ca}]$ seems to be a good abundance ratio for distinguishing dwarfs with giant planets.

Acknowledgements. R. da Silva thanks the Instituto Nacional de Pesquisas Espaciais (INPE) for its financial support in the form of a grant (PCI-DA 300.422/2011-3, MCTI/INPE/CNPq). We thank Jorge Meléndez for critically reading the manuscript. This research made use of the SIMBAD database, operated at the CDS, Strasbourg, France.

\section{References}

Adibekyan, V. Zh., Santos, N. C., Sousa, S. G., et al. 2012a, A\&A, 543, A89 Adibekyan, V. Zh., Sousa, S. G., Santos, N. C., et al. 2012b, A\&A, 545, A32
Adibekyan, V. Zh., González Hernández, J. I., Delgado Mena, E., et al. 2014, A\&A, 564, L15

Anders, E., \& Grevesse, N. 1989, Geochim. Cosmochim. Acta, 53, 197

Baranne, A., Queloz, D., Mayor, M., et al. 1996, A\&AS, 119, 373

Bertran de Lis, S., Delgado Mena, E., Adibekyan, V. Zh., Santos, N. C., \& Sousa, S. G. 2015, A\&A, 576, A89

Bond, J. C., Tinney, C. G., Butler, R. P., et al. 2006, MNRAS, 370, 163

Breiman, L., Friedman, J. H., Olshen, R. A., \& Stone, C. J. 1984, Classification and regression trees (Chapman and Hall/CRC)

Brugamyer, E., Dodson-Robinson, S. E., Cochran, W. D., \& Sneden, C. 2011, ApJ, 738, 97

Bubar, E. J., \& King, J. R. 2010, AJ, 140, 293

Chromey, F. R., Faber, S. M., Wood, A., \& Danziger, I. J. 1969, ApJ, 158, 599

da Silva, R., Milone, A., \& Reddy, B. E. 2011, A\&A, 526, A71

Dehnen, W., \& Binney, J. J. 1998, MNRAS, 298, 387

Delgado Mena, E. D., Israelian, G., González Hernández, J. I., et al. 2010, ApJ, 725,2349

Díaz-Cordovés, J., Claret, A., \& Gimenéz, A. 1995, A\&AS, 110, 329

Ecuvillon, A., Israelian, G., Santos, N. C., et al. 2004, A\&A, 418, 703

Ecuvillon. A., Israelian, G., Santos, N. C., et al. 2006, A\&A, 445, 633

Eggen, O. J. 1950, ApJ, 111, 65

ESA 1997, The Hipparcos and Tycho Catalogues, ESA SP-1200

Fischer, D. A., \& Valenti, J. 2005, ApJ, 622, 1102

Flower, P. J. 1996, ApJ, 469, 355

Gilli, G., Israelian, G., Ecuvillon, A., Santos, N. C., \& Mayor, M. 2006, A\&A, 449,723

Gonzalez, G. 2006, PASP, 118, 1494

Gonzalez, G., \& Laws, C. 2007, MNRAS, 378, 1141 
González Hernández, J. I., Israelian, G., Santos, N. C., et al. 2010, ApJ, 720, 1592

González Hernández, J. I., Delgado Mena, E., Sousa, S. G., et al. 2013a, Astron. Nachr., 334, 172

González Hernández, J. I., Delgado Mena, E., Sousa, S. G., et al. 2013b, A\&A, 552 , A6

Guenther, D. B., \& Demarque, P. 1997, ApJ, 484, 937

Holmberg, J., Nordström, B., \& Andersen, J. 2007, A\&A, 475, 519

Iben, I., Jr. 1991, ApJS, 76, 55

Johnson, D. R. H., \& Soderblom, D. R. 1987, AJ, 93, 864

Kang, W., Lee, S.-G., \& Kim, K.-M. 2011, ApJ, 736, 87

Kim, Y.-C., Demarque, P., Yi, S. K., \& Alexander, D. R. 2002, ApJS, 143, 499

Komatsu, E., Smith, K. M., Dunkley, J., et al. 2011, ApJS, 192, 18

Korotin, S., Mishenina, T., Gorbaneva, T., \& Soubiran, C. 2011, MNRAS, 415, 2093

Kupka, F., Piskunov, N. E., Ryabchikova, T. A., Stempels, H. C., \& Weiss, W. W. 1999, A\&AS, 138, 119

Kupka, F., Ryabchikova, T. A., Piskunov, N. E., Stempels, H. C., \& Weiss, W. W. 2000, Baltic Astron., 9, 590

Kurucz, R. 1993, CD-ROM No. 13, ATLAS 9 Stellar Atmosphere Programs and $2 \mathrm{~km} \mathrm{~s}^{-1}$ Grid (Cambridge, Mass.: Smithsonian Astrophysical Observatory)

Kurucz, R. 1995, CD-ROM No. 18, An Atomic and Molecular Data Bank for Stellar Spectroscopy, ASP Conf., 81

Kurucz, R. L., Furenlid, I., Brault, J., \& Testerman, L. 1984, in The Solar Flux Atlas from $296 \mathrm{~nm}$ to $1300 \mathrm{~nm}$ (National Solar Observatory)

Liu, Y., Sato, B., \& Zhao, G. 2010, PASJ, 62, 1071

Lodders, K., Palme, H., \& Gail, H.-P. 2009, In Landolt-Börnstein, New Series, Vol. VI/4B, Chap. 4.4, ed. J. E. Trümper (Berlin, Heidelberg, New York: Springer-Verlag), 560

Mata Sánchez, D., González Hernández, J. I., Israelian, G., et al. 2014, A\&A, 566, A 83

Meléndez, J., Asplund, M., Gustafsson, B., \& Yong, D. 2009, ApJ, 704, L66

Montes, D., López-Santiago, J., Gálvez, M. C., et al. 2001, MNRAS, 328, 45
Moore, C. E., Minnaert, M., \& Houtgast, J. 1966, in The Solar Spectrum 2935 A to $8770 \AA$ Å, Nat. Bur. Std. Monograph, 61

Moultaka, J., Ilovaisky, S. A., Prugniel, P., \& Soubiran, C. 2004, PASP, 116, 693 Neves, V., Santos, N. C., Sousa, S. G., Correia, A. C. M., \& Israelian, G. 2009, A\&A, 497, 563

Petigura, E. A., \& Marcy, G. W. 2011, ApJ, 735, 41

Piskunov, N. E., Kupka, F., Ryabchikova, T. A., Weiss, W. W., \& Jeffery, C. S. 1995, A\&AS, 112, 525

Ramírez, I., Meléndez, J., \& Asplund, M. 2009, A\&A, 508, L17

Ramírez, I., Asplund, M., Baumann, P., Meléndez, J., \& Bensby, T. 2010, A\&A, 521, A33

Reddy, B. E., Lambert, D. L., \& Prieto, C. A. 2006, MNRAS, 367, 1329

Robinson, S. E., Laughlin, G., Bodenheimer, P., \& Fischer, D. 2006, ApJ, 643, 484

Ryabchikova, T. A., Piskunov, N. E., Kupka, F., \& Weiss, W. W. 1997, Baltic Astron., 6, 244

Sandage, A., \& Fouts, G. 1986, AJ, 91, 1140

Santos, N. C., Israelian, G., \& Mayor, M. 2001, A\&A, 373, 1019

Santos, N. C., Israelian, G., \& Mayor, M. 2004, A\&A, 415, 1153

Smiljanic, R., Porto de Mello, G. F., \& da Silva, L. 2007, A\&A, 468, 679

Smith, V. V., Cunha, K., \& Lazzaro, D. 2001, AJ, 121, 3207

Sneden, C. 2002, http://verdi.as.utexas.edu/moog.html

Sousa, S. G., Santos, N. C., Israelian, G., Mayor, M., \& Monteiro, J. P. F. G. 2007, A\&A, 469, 783

Steffen, M. 1985, A\&AS, 59, 403

Takeda, Y., Sato, B., \& Murata, D. 2008, PASJ, 60, 781

Torres, G. 2010, AJ, 140, 1158

van Leeuwen, F. 2007, Hipparcos, the New Reduction of the Raw Data, Astrophys. Space Sci. Lib., 350

Wallace, L., Hinkle, K. H., Livingston, W. C., \& Davis, S. P. 2011, ApJS, 195, 6

Williams, P. M. 1971, The Observatory, 91, 37

Yi, S., Demarque, P., Kim, Y.-C., et al. 2001, ApJS, 136, 417

Zăcs, L. 1994, A\&A, 283, 937 